\title{
Nematicity and Magnetism in FeSe and Other Families of Fe-Based Superconductors
}

\author{
Youichi Yamakawa, ${ }^{1}$ Seiichiro Onari, ${ }^{2}$ and Hiroshi Kontani ${ }^{1}$ \\ ${ }^{1}$ Department of Physics, Nagoya University, Furo-cho, Nagoya 464-8602, Japan \\ ${ }^{2}$ Department of Physics, Okayama University, Okayama 700-8530, Japan
}

(Received 25 October 2015; revised manuscript received 3 May 2016; published 3 June 2016)

\begin{abstract}
Nematicity and magnetism are two key features in Fe-based superconductors, and their interplay is one of the most important unsolved problems. In FeSe, the magnetic order is absent below the structural transition temperature $T_{\text {str }}=90 \mathrm{~K}$, in stark contrast to the fact that the magnetism emerges slightly below $T_{\text {str }}$ in other families. To understand such amazing material dependence, we investigate the spinfluctuation-mediated orbital order $\left(n_{x z} \neq n_{y z}\right)$ by focusing on the orbital-spin interplay driven by the strong-coupling effect, called the vertex correction. This orbital-spin interplay is very strong in FeSe because of the small ratio between the Hund's and Coulomb interactions $(\bar{J} / \bar{U})$ and large $d_{x z}, d_{y z}$-orbital weight at the Fermi level. For this reason, in the FeSe model, the orbital order is established irrespective of the fact that the spin fluctuations are very weak, so the magnetism is absent below $T_{\text {str. }}$. In contrast, in the $\mathrm{LaFeAsO}$ model, the magnetic order appears just below $T_{\text {str }}$ both experimentally and theoretically. Thus, the orbital-spin interplay due to the vertex correction is the key ingredient in understanding the rich phase diagram with nematicity and magnetism in Fe-based superconductors in a unified way.
\end{abstract}

DOI: $10.1103 /$ PhysRevX.6.021032

\section{INTRODUCTION}

In Fe-based superconductors, the origin of the electronic nematic state and its relation to the magnetism have been a central unsolved problem. Recently, the nonmagnetic nematic state in FeSe has attracted increasing attention as a key to solve the origin of the nematicity. FeSe undergoes structural and superconducting transitions at $T_{\text {str }}=90 \mathrm{~K}$ and $T_{c}=9 \mathrm{~K}$, respectively, whereas the magnetic transition is absent down to $0 \mathrm{~K}$ [1]. The strength of the low-energy antiferromagnetic (AFM) fluctuations is very weak above $T_{\text {str }}$, while it starts to increase below $T_{\text {str }}$ [2-7]. In stark contrast, the magnetic transition occurs at $T_{\text {mag }}$ slightly below $T_{\text {str }}$ in other undoped Fe-based superconductors. Since the relation $T_{\text {str }}>T_{\text {mag }}$ is unable to be explained by the random-phase approximation (RPA), we should develop the microscopic theory beyond the mean-field-level approximations.

Up to now, two promising triggers for the structure transition have been discussed intensively: In the spinnematic scenario [8-12], the trigger is the spin-nematic order. This spin-fluctuation induced spin-quadrupole order could emerge above $T_{\text {mag }}$ in highly magnetically frustrated systems. In the orbital order scenario [13-16], the trigger is the ferro-orbital (FO) order $n_{x z} \neq n_{y z}$. Above $T_{\text {str }}$, the

Published by the American Physical Society under the terms of the Creative Commons Attribution 3.0 License. Further distribution of this work must maintain attribution to the author(s) and the published article's title, journal citation, and DOI.
Subject Areas: Condensed Matter Physics, Strongly Correlated Materials strong orbital or spin-nematic fluctuations are observed by the measurements of shear modulus $C_{66}[2,17,18]$, Raman spectroscopy [19-22], and in-plane resistivity anisotropy $[23,24]$. The nematic orbital fluctuations originate from the strong orbital-spin mode coupling due to the strongcoupling effect, which is described by the AslamazovLarkin vertex correction (AL-VC). The electronic nematic state studied in single-orbital models [25] is more easily realized in multiorbital systems thanks to the AL-VC mechanism [16].

Except for the presence or absence of magnetism below $T_{\text {str }}$, FeSe and other Fe-based superconductors show common electronic properties. Below $T_{\text {str }}$, in both FeSe and $\mathrm{BaFe}_{2} \mathrm{As}_{2}$, large orbital polarization $\Delta E \equiv E_{y z}-E_{x z} \sim$ $50 \mathrm{meV}$ [26-33] is observed. Such large $\Delta E$ originates from the electron-electron correlation since the lattice distortion $(a-b) /(a+b)$ is just $0.2 \%-0.3 \%$, as we discuss based on band calculation in Appendix A. Above $T_{\text {str }}$, the electronic nematic susceptibility is enhanced in both $\mathrm{BaFe}_{2} \mathrm{As}_{2}$ $[17,19,23]$ and FeSe [2,18], following the similar CurieWeiss behavior. These facts indicate that the common microscopic mechanism drives the nematic order and fluctuations in all Fe-based superconductors, in spite of the presence or absence of the magnetism.

The realistic multiorbital Hubbard models for Fe-based superconductors, which are indispensable for the present study, were derived by using the first-principles method in Ref. [34]. To understand the absence of the magnetism below $T_{\text {str }}$ in $\mathrm{FeSe}$, one significant hint is the smallness of the ratio between the Hund's and Coulomb interactions, $\bar{J} / \bar{U}$, since the Hund's coupling enlarges (suppresses) the 
intrasite magnetic (orbital) polarization, which is verified by the functional renormalization-group (fRG) theory $[35,36]$. Another significant hint is the absence of the $d_{x y}$-orbital hole pocket in FeSe, which is favorable for the orbital-spin interplay on the $\left(d_{x z}, d_{y z}\right)$ orbitals due to the AL-VC mechanism.

The goal of this paper is to explain the amazing variety of the electronic nematic states in Fe-based superconductors, especially the nonmagnetic nematic state in FeSe, on the same footing microscopically. For this purpose, we study the spin-fluctuation-mediated orbital order by applying the self-consistent vertex-correction (SC-VC) method [16] to the first-principles models. In FeSe, the orbital-spin interplay is significant because of the smallness of $\bar{J} / \bar{U}$ and the absence of a $d_{x y}$-hole pocket. For this reason, the orbital order is realized even when the spin fluctuations are substantially weak. The rich variety of the phase diagrams in Fe-based superconductors, such as the presence or absence of the magnetic order in the nematic phase, are well understood by analyzing the vertex correction seriously. The SC-VC theory had been successfully applied to explain the phase diagram in $\operatorname{LaFeAs}(\mathrm{O}, \mathrm{H})$ [37], nematic CDW in cuprates $[38,39]$, and triplet superconductivity in $\mathrm{Sr}_{2} \mathrm{RuO}_{4}$ [36].

We comment that the localized spin models have been successfully applied to the nematic order, stripe magnetic order, and so on [40]. On the other hand, weak-coupling theories have also been satisfactorily applied to Fe-based superconductors [41]. In the present work, we study the mechanisms of the nematicity and magnetism in various Fe-based superconductors in terms of the itinerant picture, by taking the strong-coupling effect due to the $\mathrm{AL}-\mathrm{VC}$ into account. The significant role of the AL-VC on the orbital fluctuations has been confirmed by the fRG theory $[35,36]$. The AL-VC is important to reproduce the KugelKhomskii-type orbital-spin interaction [16].

\section{MODEL HAMILTONIAN AND SC-VC THEORY}

Here, we study the realistic $d-p$ Hubbard models

$$
H_{\mathrm{M}}(r)=H_{\mathrm{M}}^{0}+r H_{\mathrm{M}}^{U}
$$

for $\mathrm{M}=\mathrm{LaFeAsO}$ and $\mathrm{FeSe}$ by applying the SC-VC method [16]. In Eq. (1),

$$
H_{\mathrm{M}}^{0}=\sum_{\boldsymbol{k}, l m, \sigma} c_{\boldsymbol{k}, l \sigma}^{\dagger} h_{\mathrm{M}, l m}^{0}(\boldsymbol{k}) c_{\boldsymbol{k}, m \sigma}
$$

is the 8-orbital $d$-p tight-binding (TB) model in $\boldsymbol{k}$ space, which is obtained by using the WIEN2k and WANNIER90 software; see Appendix A for a detailed explanation. Here, $\sigma$ is the spin index, and $l, m$ are the orbital indices: Hereafter, we denote the five $d$ orbitals $d_{3 z^{2}-r^{2}}, d_{x z}, d_{y z}, d_{x y}$, $d_{x^{2}-y^{2}}$ as $1,2,3,4,5$, and three $p$ orbitals as $6-8$. The band structure and Fermi surfaces (FSs) in the LaFeAsO model are shown in Figs. 1(a) and 1(b), respectively. Similar FSs with three holelike FSs (h-FSs) and two electronlike FSs (e-FSs) exist in many Fe-based superconductors. In FeSe, however, h-FS3 composed of a $d_{x y}$ orbital is absent, and the size of each FS is very small as clarified by the ARPES $[27,32,33]$ and dHvA $[42,43]$ studies. To reproduce the experimental band structure of FeSe, we introduce the additional intra-orbital hopping parameters into $H_{\mathrm{FeSe}}^{0}$, in order to shift the $d_{x y}$-orbital band $\left(d_{x z / y z}\right.$-orbital band) at $(\Gamma, \mathrm{M}, \mathrm{X})$ points by $(0,-0.25,+0.24)[(-0.24,0,+0.12)]$ in units of eV; see Appendix A. These energy shifts might be induced by the self-energy [44]. The constructed FSs in the FeSe model are shown in Fig. 1(c). Since each Fermi pocket is very shallow, the superconductivity in FeSe could be close to a BCS-BEC crossover [45].

In Eq. (1), $H_{\mathrm{M}}^{U}$ is the first-principles screened Coulomb potential for $d$ orbitals given by the "constrained-RPA method" [34] given as

$$
\begin{aligned}
H_{\mathrm{M}}^{U}= & \frac{1}{2} \sum_{i, l, m, \sigma, \sigma^{\prime}}\left\{U_{m, l} n_{i, l \sigma} n_{i, m, \sigma^{\prime}}\left(1-\delta_{l, m} \delta_{\sigma,-\sigma^{\prime}}\right)\right. \\
& \left.+J_{m, l} c_{i, m \sigma}^{\dagger} c_{i, l \sigma}\left(c_{i, l \sigma^{\prime}}^{\dagger} c_{i, m \sigma^{\prime}}+c_{i, m \sigma^{\prime}}^{\dagger} c_{i, l \sigma^{\prime}} \delta_{\sigma,-\sigma^{\prime}}\right)\right\},
\end{aligned}
$$
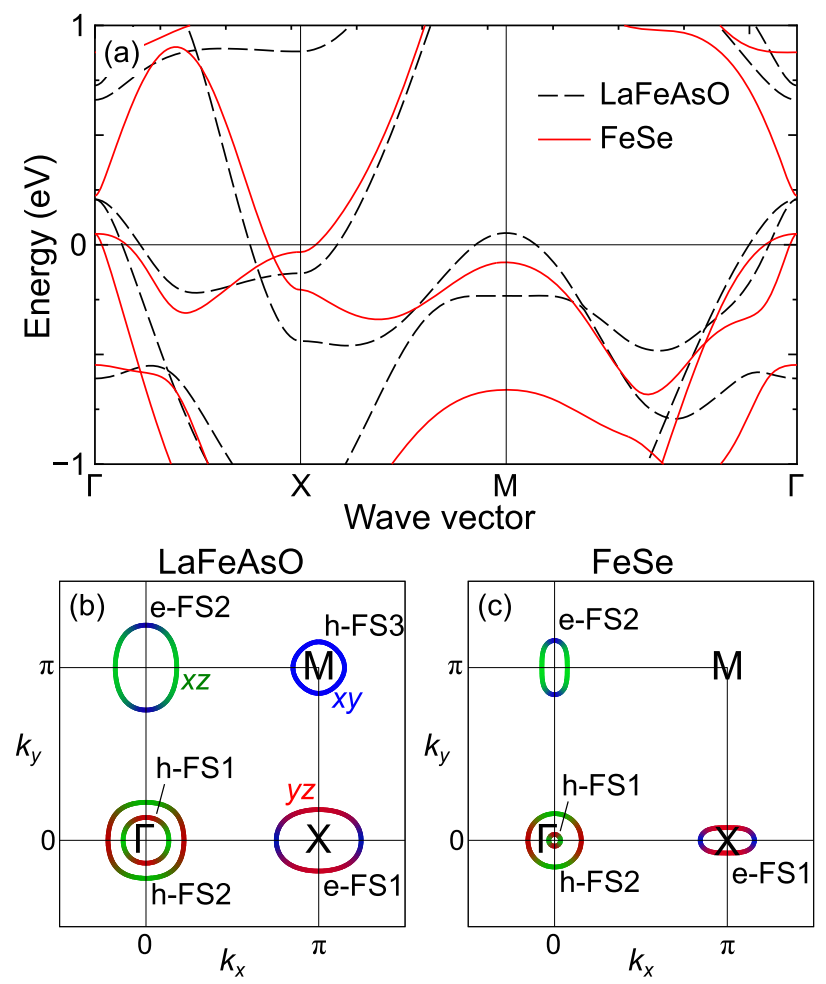

FIG. 1. (a) Band structures of the eight-orbital TB models for $\mathrm{LaFeAsO}$ and FeSe. (b) FSs for the LaFeAsO TB model. (c) FSs for the FeSe TB model. The colors correspond to 2 (green), 3 (red), and 4 (blue), respectively. 
where $U_{m, l}$ and $J_{m, l}$ are orbital-dependent Coulomb and Hund's interactions for $d$ electrons, respectively [34]. The averaged intra-orbital Coulomb interaction $\bar{U} \equiv \frac{1}{5} \sum_{l=1}^{5} U_{l, l}$ and Hund's interactions $\bar{J} \equiv \frac{1}{10} \sum_{l>m} J_{l, m}$ are $(\bar{U}, \bar{J})=(7.21,0.681)$ for $\mathrm{FeSe}$, and $(\bar{U}, \bar{J})=$ $(4.23,0.568)$ for LaFeAsO in units of $\mathrm{eV}$ [34]. Thus, the ratio $\bar{J} / \bar{U}=0.0945$ in FeSe is much smaller than the ratio $\bar{J} / \bar{U}=0.134$ in $\mathrm{LaFeAsO}$. Such strong material dependence of $(\bar{U}, \bar{J})$ is understood as follows: $U_{l, m}$ is strongly screened by the screening bands (excluding the eight bands in $H_{\mathrm{M}}^{0}$ ), whereas the screening of $J_{l, m}$ is much weaker and the number of the screening bands is small in FeSe [46]. The factor $r(<1)$ in Eq. (1) is introduced to adjust the spin fluctuation strength. The ratio $J_{l, m} / U_{l, m}$ is unchanged by introducing the factor $r[47,48]$.

The $8 \times 8$ Green function in the orbital basis is given as

$$
\hat{G}(k)=\left[\hat{z}^{-1} i \epsilon_{n}+\mu-\hat{h}_{\mathrm{M}}^{0}(\boldsymbol{k})\right]^{-1},
$$

where $k=\left(\boldsymbol{k}, \epsilon_{n}=(2 n+1) \pi T\right), \hat{h}_{\mathrm{M}}^{0}(\boldsymbol{k})$ is the kinetic term in Eq. (2), and $\hat{z}^{-1} \equiv 1-\partial \hat{\Sigma} /\left.\partial \epsilon\right|_{\epsilon=0}$ represents the mass enhancement due to the self-energy at the Fermi level. Here, we introduce the constant mass-enhancement factor for the $d$ orbital $1 / z_{l}(\geq 1)$. Then, Eq. (4) gives the coherent part of the Green function, which mainly determines the low-energy electronic properties. In the present study, $r$ and $z_{l}$ are the fitting parameters. In FeSe, the orbital order is obtained in the real first-principles Hamiltonian $(r \approx 1)$ by taking the experimental mass-enhancement factors $z_{l}^{-1} \approx 4$ into account, as shown later.

The $d$-orbital charge (spin) susceptibilities (per spin) is given in the following $5^{2} \times 5^{2}$ matrix form:

$$
\hat{\chi}^{c(s)}(\boldsymbol{q})=\hat{\Phi}^{c(s)}(\boldsymbol{q})\left[1-\hat{\Gamma}^{c(s)} \hat{\Phi}^{c(s)}(\boldsymbol{q})\right]^{-1},
$$

where $\hat{\Phi}^{c(s)}(\boldsymbol{q})=\hat{\chi}^{0}(\boldsymbol{q})+\hat{X}^{c(s)}(\boldsymbol{q})$ is the irreducible susceptibility for the charge (spin) channel. In the SC-VC theory, we employ the AL-VC as $\hat{X}^{c, s}(\boldsymbol{q})$ and perform the self-consistent calculation with respect to the AL-VC and susceptibilities. Using the Green function in Eq. (4), the bare susceptibility is

$$
\chi_{l, l^{\prime} ; m, m^{\prime}}^{0}(q)=-T \sum_{k} G_{l, m}(k+q) G_{m^{\prime}, l^{\prime}}(k),
$$

where $q=\left(\boldsymbol{q}, \omega_{l}=2 l \pi T\right)$. Also, the AL-VC for the charge susceptibility is given as

$$
\begin{aligned}
X_{l, l^{\prime} ; m, m^{\prime}}^{\mathrm{AL}, c}(q)= & \frac{T}{2} \sum_{p} \sum_{a \sim h} \Lambda_{l, l^{\prime} ; a, b ; e, f}(q ; p)\left\{3 V_{a, b ; c, d}^{s}(p+q)\right. \\
& \left.\times V_{e, f ; g, h}^{s}(-p)+V_{a, b ; c, d}^{c}(p+q) V_{e, f ; g, h}^{c}(-p)\right\} \\
& \times \Lambda_{m, m^{\prime} ; c, d ; g, h^{\prime}}(q ; p),
\end{aligned}
$$

where $p=\left(\boldsymbol{p}, \omega_{m}\right)$, and $\hat{V}^{s, c}(q) \equiv \hat{\Gamma}^{s, c}+\hat{\Gamma}^{s, c} \hat{\chi}^{s, c}(q) \hat{\Gamma}^{s, c}$. The three-point vertex $\hat{\Lambda}(q ; p)$, which gives the coupling between two magnons and one orbiton, is given as

$$
\begin{aligned}
& \Lambda_{l, l^{\prime} ; a, b ; e, f}(q ; p) \\
& \quad=-T \sum_{k^{\prime}} G_{l, a}\left(k^{\prime}+q\right) G_{f, l^{\prime}}\left(k^{\prime}\right) G_{b, e}\left(k^{\prime}-p\right),
\end{aligned}
$$

and $\quad \Lambda_{m, m^{\prime} ; c, d ; g, h}^{\prime}(q ; p) \equiv \Lambda_{c, h ; m, g ; d, m^{\prime}}(q ; p)+\Lambda_{g, d ; m, c ; h, m^{\prime}}$ $(q ;-p-q)$. We stress that the strong temperature dependence of the three-point vertex is significant for realizing the orbital order. We include all $U^{2}$ terms without the double counting in order to obtain quantitatively reliable results. Equation (7) means that the charge AL-VC becomes large in the presence of strong spin fluctuations. More detailed explanations are presented in the textbook [49].

In the present study, we neglected the spin-channel VCs since they are expected to be unimportant as discussed in Ref. [50]. In Appendix B, we verify the validity of this simplification in the present model by performing a timeconsuming self-consistent calculation with respect to both charge- and spin-channel Maki-Thompson (MT) and AL-VCs.

Hereafter, we mainly discuss the total spin susceptibility, $\chi^{s}(\boldsymbol{q}) \equiv \sum_{l, m} \chi_{l, l ; m, m}^{s}(\boldsymbol{q})$, and the orbital susceptibilities for $O_{x^{2}-y^{2}}=n_{x z}-n_{y z}, \quad \chi_{x^{2}-y^{2}}^{c}(\boldsymbol{q}) \equiv \chi_{2,2 ; 2 ; 2}^{c}(\boldsymbol{q})+\chi_{3,3 ; 3,3}^{c}(\boldsymbol{q})-$ $2 \chi_{2,2 ; 3,3}^{c}(\boldsymbol{q})$. The divergence of $\chi_{x^{2}-y^{2}}^{c}(\boldsymbol{q})$ at $\boldsymbol{q}=\mathbf{0}$ gives rise to the FO order $n_{x z} \neq n_{y z}$. The charge (spin) Stoner factor $\alpha_{C(S)}$ is given by the maximum eigenvalue of $\hat{\Gamma}^{c(s)} \hat{\Phi}^{c(s)}(\boldsymbol{q})$ in Eq. (5), and the charge (spin) susceptibility is enlarged in proportion to the charge (spin) Stoner enhancement factor $S_{C(S)} \equiv\left(1-\alpha_{C(S)}\right)^{-1}$.

As explained in Ref. [50], the development of $\chi_{x^{2}-y^{2}}^{c}(\mathbf{0})$ is mainly induced by the diagonal elements of $\hat{\Phi}^{c}$ with respect to $l=2,3$. If we drop the off-diagonal elements of $\hat{\Phi}^{c}$ approximately, $\chi_{x^{2}-y^{2}}^{c}(\mathbf{0})$ is given as

$$
\chi_{x^{2}-y^{2}}^{c}(\mathbf{0}) \approx 2 \Phi^{c} /\left(1-(1-5 J / U) U \Phi^{c}\right),
$$

where $U \equiv U_{2,2}=U_{3,3}, J \equiv J_{2,3}, \Phi^{c} \equiv \chi_{l, l i l, l}^{0}(\mathbf{0})+X_{l, l i l, l}^{c}(\mathbf{0})$ ( $l=2$ or 3 ). Thus, the charge Stoner factor is $\alpha_{C}=$ $(1-5 J / U) U \Phi^{c} \approx(1-5 J / U)\left(1+U X^{c}\right)$, considering the relation $\chi^{0}(\boldsymbol{q}) \approx 1 / U$. Within the $\operatorname{RPA}\left(\Phi^{c}=\Phi^{s}=\chi^{0}\right)$, only the spin fluctuations develop since the relation $\alpha_{S}>\alpha_{C}$ is satisfied for $J>0$. However, the opposite relation $\alpha_{C}>\alpha_{S}$ is realized if the relation $\hat{\Phi}^{c} \gg \chi^{0}$ is satisfied because of the charge-channel AL-VC [37].

\section{NUMERICAL RESULTS FOR LaFeAsO AND FeSe}

First, we analyze the $\mathrm{LaFeAsO}$ model based on the SC-VC theory. For $z=1$ for each $l$, the obtained $\chi^{s}(\boldsymbol{q})$ and 


\section{LaFeAsO}
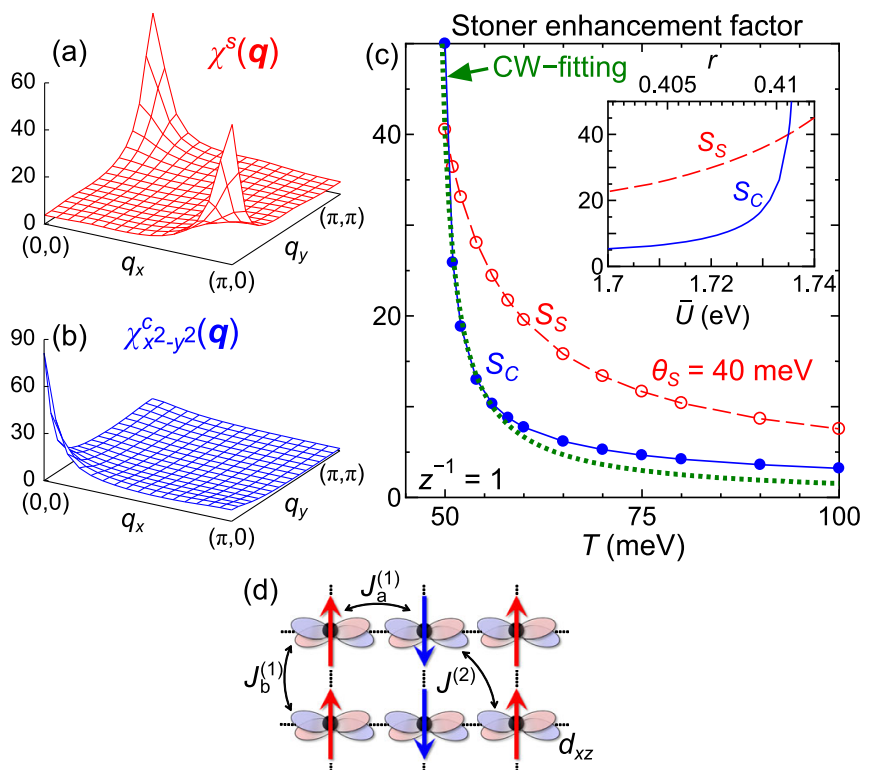

FIG. 2. (a) $\chi^{s}(\boldsymbol{q})$ and (b) $\chi_{x^{2}-y^{2}}^{c}(\boldsymbol{q})$ obtained by the SC-VC method for $\mathrm{LaFeAsO}(z=1)$. Note that other FO susceptibility $\left[\chi_{z^{2}}^{c}(\boldsymbol{q})\right]$ and antiferro-orbital susceptibilities $\left[\chi_{x z / y z}^{c}(\boldsymbol{q})\right]$ also develop secondarily. (c) Orbital (spin) enhancement factor $S_{C(S)}$ as a function of $T$ for $\mathrm{LaFeAsO}(z=1)$ at $r=0.41$. The charge and spin Weiss temperatures are $\theta_{C}=48 \mathrm{meV}$ and $\theta_{S}=40 \mathrm{meV}$, respectively. Inset: $S_{C(S)}$ as a function of $r$ at $T=50 \mathrm{meV}$. Note that $\bar{U}=$ $4.23 r \mathrm{eV}$ for $\mathrm{LaFeAsO}$. (d) Orbital-spin interplay in the localized $\left(d_{x z}, d_{y z}\right)$-orbital model, known as the Kugel-Khomskii coupling.

$\chi_{x^{2}-y^{2}}^{c}(\boldsymbol{q})$ are shown in Figs. 2(a) and 2(b), respectively, for $r=0.41(\bar{U}=1.74 \mathrm{eV})$ at $T=50 \mathrm{meV}$. Here, the number of $\boldsymbol{k}$ meshes is $32 \times 32$, and the number of Matsubara frequencies is 256. Thus, both AFM and FO susceptibilities develop divergently, and the realized enhancement factors are $S_{S} \approx 40$ and $S_{C} \approx 50$. The $r$ dependence of the enhancement factors at $T=50 \mathrm{meV}$ is shown in the inset of Fig. 2(c): Both $S_{S}$ and $S_{C}$ increase with $r$, and they are equivalent at $r^{*}=0.41$. The lower the temperature is, the smaller $r^{*}$ is, whereas the value of $S_{S}=S_{C}$ at $r^{*}$ is approximately independent of $T$. A similar result is obtained in the $\mathrm{BaFe}_{2} \mathrm{As}_{2}$ model as shown in Appendix C.

In addition, other antiferro-orbital susceptibilities $\chi_{x z}^{c}(\boldsymbol{q})=2\left[\chi_{3,4 ; 3,4}^{c}(\boldsymbol{q})+\chi_{3,4 ; 4,3}^{c}(\boldsymbol{q})\right] \quad$ and $\quad \chi_{y z}^{c}(\boldsymbol{q})=$ $2\left[\chi_{2,4 ; 2,4}^{c}(\boldsymbol{q})+\chi_{2,4 ; 4,2}^{c}(\boldsymbol{q})\right]$ also develop moderately as reported in previous studies $[16,37,49]$. The obtained results are essentially similar to the results obtained in the five $d$-orbital Hubbard model for $\mathrm{LaFeAsO}$, as explained in Ref. [16].

Figure 2(c) shows the temperature dependence of the Stoner enhancement factors at $r=0.41$. Both $S_{C}$ and $S_{S}$ approximately follow the Curie-Weiss behaviors with the charge and spin Weiss temperatures $\theta_{C}=48 \mathrm{meV}$ and $\theta_{S}=40 \mathrm{meV}$, respectively. The obtained relation $\chi_{x^{2}-y^{2}}^{c}(\mathbf{0}) \propto\left(T-\theta_{C}\right)^{-1}$ is consistent with the CurieWeiss behavior of the nematic susceptibilities in $\mathrm{BaFe}_{2} \mathrm{As}_{2}$, derived from $C_{66}[17,18]$, Raman spectroscopy $[19,20]$, and in-plane resistivity anisotropy [23]. Since $\theta_{C} \sim \theta_{S}$, one could interpret that the orbital order in $\mathrm{LaFeAsO}$ is driven by the spin fluctuations.

The orbital-spin interplay due to the AL-VC is intuitively understood in terms of the strong-coupling picture $U \gg W_{\text {band }}$ [37]: As shown in Fig. 2(d), when the FO order $n_{x z} \gg n_{y z}$ is realized, the nearest-neighbor exchange interaction has large anisotropy $J_{a}^{(1)} \gg J_{b}^{(1)}$. Then, the stripe AFM order with $\boldsymbol{Q}=(\pi, 0)$ appears if $\boldsymbol{J}^{(2)}$ is not too small. Thus, the FO order/fluctuations and AFM order/fluctuations emerge cooperatively in the localized model, and such Kugel-Khomskii-type orbital-spin interplay is explained by the AL-VC in the weak-coupling picture.

Next, we analyze the FeSe model, in which the ratio $\bar{J} / \bar{U}$ is considerably small. In FeSe, the experimental massenhancement factor is about 10 for the $d_{x y}$ orbital, and 3-4 for other $d$ orbitals according to the ARPES study [27]. Therefore, we put $z_{l}^{-1}=z^{-1}$ for $l \neq 4$ and $z_{4}^{-1}=3 z^{-1}$ in the present study. We find that the peak of $\chi^{s}(\boldsymbol{q})$ moves from $\boldsymbol{q}=(\pi, \pi)$ to the experimental peak position $\boldsymbol{q}=(\pi, 0)$ [4-7] for $z_{4}^{-1} \geq 1.5 z^{-1}$, and the results of the SC-VC method are essentially unchanged for $z_{4}^{-1} \geq 1.5 z^{-1}$. Figures 3(a) and 3(b) show the obtained $\chi^{s}(\boldsymbol{q})$ and $\chi_{x^{2}-y^{2}}^{c}(\boldsymbol{q})$ for $r=0.25(\bar{U}=1.76 \mathrm{eV})$ at $T=50 \mathrm{meV}$ in the case of $z=1$. We see that only the FO susceptibilities develop divergently $\left[S_{C} \approx 50\right]$, whereas the AFM susceptibility remains small $\left[S_{S} \approx 8\right]$, consistent with experiments for FeSe. The $r$ dependence of the Stoner enhancement factors at $T=50 \mathrm{meV}$ is shown in the inset of Fig. 3(c): With increasing $r, S_{C}$ increases rapidly whereas $S_{S}$ remains small.

Figure 3(c) shows the temperature dependence of the enhancement factors at $r=0.25$. We stress that $S_{C}$ approximately follows the Curie-Weiss behavior with the Weiss temperature $\theta_{C}=48 \mathrm{meV}$, which is consistent with the experimental Curie-Weiss behavior with positive $\theta_{C}$ in FeSe [2]. Since the spin Weiss temperature takes a large negative value $\left(\theta_{S} \sim-20 \mathrm{meV}\right)$, which is also consistent with experiments, one may consider that the orbital order in FeSe stems from causes other than spin fluctuations.

\section{ORIGIN OF THE RELATION $S_{C} \gg S_{S}$ IN FeSe}

In this section, we discuss why the relation $S_{C} \gg S_{S}$ $\left(\theta_{C}>0\right.$ and $\left.\theta_{S}<0\right)$ is realized in FeSe. First, we focus on the ratio between the Hund's and Coulomb interactions $\bar{J} / \bar{U}$. It is intuitively obvious that the ratio $\bar{J} / \bar{U}$ is an important control parameter for the orbital nematicity: For larger $\bar{J} / \bar{U}$, the local configuration of the two electrons in the $\left(d_{x z}, d_{y z}\right)$ orbitals is $\left|d_{x z}, \uparrow\right\rangle \otimes\left|d_{y z}, \uparrow\right\rangle$, where the 


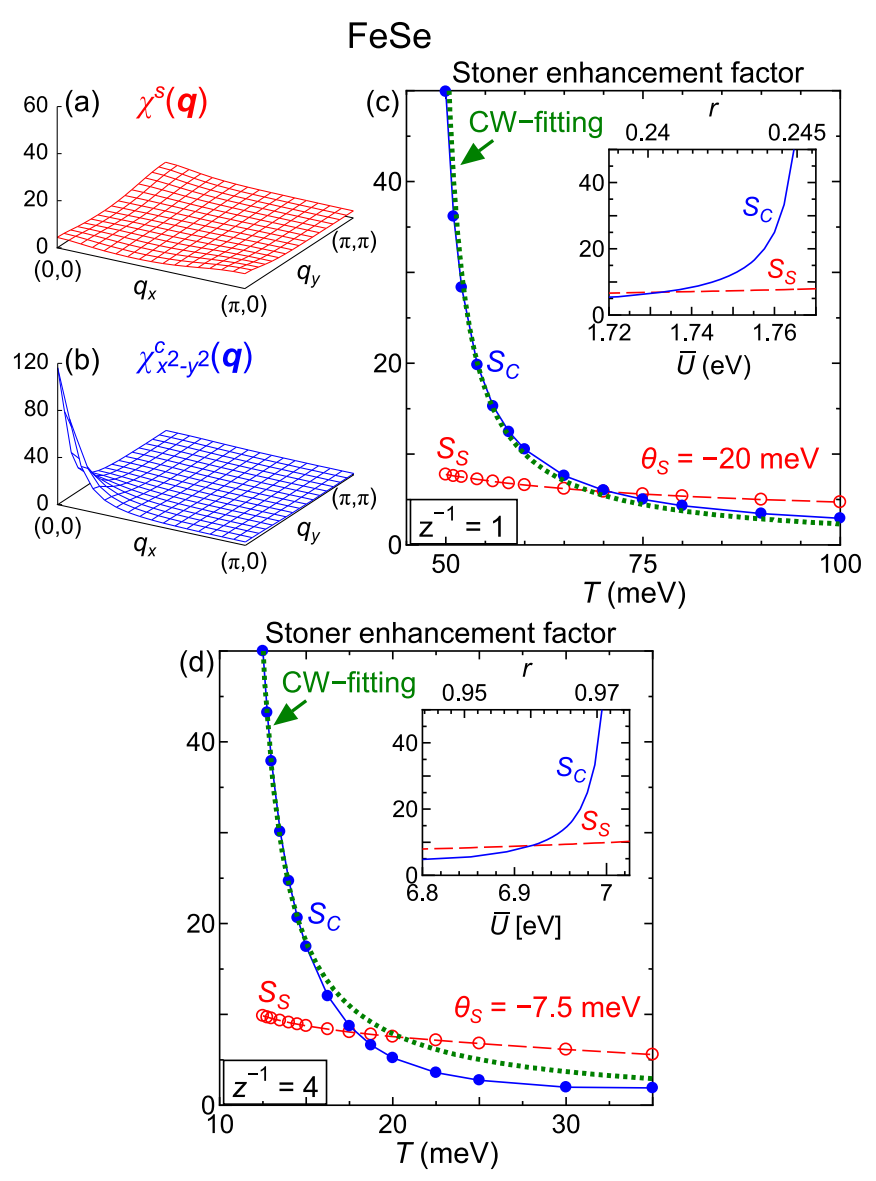

FIG. 3. (a) $\chi^{s}(\boldsymbol{q})$ and (b) $\chi_{x^{2}-y^{2}}^{c}(\boldsymbol{q})$ obtained by the SC-VC method for FeSe $(z=1)$, for $r=0.25$ at $T=50 \mathrm{meV}$. (c) $T$ dependence of the Stoner enhancement factors for $\mathrm{FeSe}(z=1)$ at $r=0.25$. Note that $\bar{U}=7.21 r \mathrm{eV}$ for FeSe. Inset: Stoner enhancement factors as a function of $r$ for $\operatorname{FeSe}(z=1)$ at $T=50 \mathrm{meV}$. (d) $T$ dependence of the enhancement factors for FeSe $\left(z^{-1}=4\right)$ at $r=0.97$. The charge and spin Weiss temperatures are $\theta_{C}=12 \mathrm{meV}$ and $\theta_{S} \sim-7.5 \mathrm{meV}$, respectively. Inset: Stoner enhancement factors as a function of $r$ for FeSe $\left(z^{-1}=4\right)$ at $T=12.5 \mathrm{meV}$.

magnetic moment is $s_{z}=1$, whereas the orbital polarization is $n_{x z}-n_{y z}=0$. Thus, the smallness of $\bar{J} / \bar{U}$ in FeSe is favorable for the emergence of the orbital order without magnetization.

Microscopically, as we discuss in Sec. II, the charge Stoner factor for $\chi_{x^{2}-y^{2}}^{c}(\mathbf{0})$ is $\alpha_{C} \approx(1-5 J / U)\left(1+U X^{c}\right)$, where $X^{c}$ is the charge AL-VC for orbital 2 or 3 at $\boldsymbol{q}=\mathbf{0}$. Since $\bar{J} / \bar{U}=0.0945$ in FeSe, the orbital order is realized by relatively small AL-VC: $X^{c} \sim 0.9 \chi^{0}(\mathbf{0})$. In LaFeAsO, in contrast, large $\mathrm{AL}-\mathrm{VC}$ of order $\sim 2 \chi^{0}(\mathbf{0})$ is required to realize the orbital order. The obtained AL-VCs in both systems are shown in Fig. 9(c) in Appendix D.

We discuss why the AL-VC is important in the FeSe model with $\theta_{S}<0$ : As we explain in Appendix D, the $T$ dependence of the AL-VC is given as $X^{c} \sim \Lambda^{2} T S_{S}$, where $\Lambda$ is the three-point vertex that represents the interference between two short-living magnons. We find the relation $\Lambda^{2} \propto T^{-a}$ with $a \approx 1$ at low temperatures due to the good nesting between h-FSs and e-FSs [20]. Thanks to the strong enhancement of $\Lambda$ at low temperatures, the orbital order $\left(\alpha_{C}=1\right)$ is realized even if $\theta_{S}$ is negative. (Note that $T S_{S}$ decreases as $T \rightarrow 0$ when $\theta_{S}<0$.) Thus, serious diagrammatic analysis of the AL-VC is necessary to understand the rich normal-state phase diagrams in Fe-based superconductors.

The enhancement of the nematic susceptibility due to the significant $T$ dependence of $\Lambda^{2}\left(\propto T^{-a}\right)$ was discussed in Refs. [20,21,51,52]. However, the reported exponent $a$ is not universal since it depends on the band structure and temperature range. In Appendix $\mathrm{E}$, we show the $T$ dependence of the three-point vertex for $\mathrm{LaFeAsO}$ and FeSe models for a wide temperature range. It is found that $a \approx 1$ for $T=20 z \sim 100 z[\mathrm{meV}]$, where $z<1$ is the bandrenormalization factor. Because of such large $T$ dependence of $a, \chi_{x^{2}-y^{2}}^{c}(\mathbf{0})$ obtained by the present study follows the Curie-Weiss law only approximately.

Finally, we stress the importance of the orbital dependence of the spin fluctuation strength. Since the $d_{x y}$ orbital h-FS (h-FS3) is absent in FeSe, the relation $\chi_{2(3)}^{s}(\boldsymbol{q}) \gg$ $\chi_{4}^{s}(\boldsymbol{q})\left(\chi_{l}^{s}(\boldsymbol{q}) \equiv \chi_{l, l i l, l}^{s}(\boldsymbol{q})\right)$ is realized. This condition is favorable for the development of $\chi_{x^{2}-y^{2}}^{c}(\mathbf{0})$ since $X_{2(3)}^{c}$ is enlarged by the spin fluctuations on the $\left(d_{x z}, d_{y z}\right)$ orbitals. A more detailed analysis is given in Appendix D.

\section{EFFECT OF THE MASS-ENHANCEMENT FACTOR}

Here, we study the effect of the mass-enhancement factor: We study the FeSe model in the case of $z^{-1}=4$ for $\left(d_{x z}, d_{y z}\right)$ orbitals. The obtained $S_{C, S}$ as functions of $r$ are shown in the inset of Fig. 3(d) at $T=12.5 \mathrm{meV}$. Here, $S_{S}$ remains small even for $r \sim 1$ since the bare susceptibility is suppressed by $z$. In contrast, $S_{C}$ is enlarged to 50 for $r \approx 0.97$, which is very close to the exact first-principles Hubbard model $H_{\mathrm{FeSe}}(r=1)$. The $T$ dependence of $S_{C, S}$ is shown in Fig. 3(d): Good Curie-Weiss behavior with $\theta_{C}=$ $12 \mathrm{meV}$ is obtained by putting $r=0.97$.

To understand the similarity between the results in Fig. 3(c) for $z=1$ and the results in Fig. 3(d) for $z^{-1}=4$, we prove that both $\alpha_{C}$ and $\alpha_{S}$ are independent of $z$ under the rescaling $T \rightarrow z T$ and $(U, J) \rightarrow(U, J) / z$. Here, we assume that $z_{l}^{-1}=z^{-1}$, and we neglect the $T$ dependence of $\mu$ for simplicity. Under the scaling $T \rightarrow z T$, the Green function $\hat{G}(\boldsymbol{k}, n)$ at Matsubara integer $n$ given in Eq. (4) is independent of $z$. For this reason, the bare susceptibility $\chi^{0}(\boldsymbol{q})=-T \sum_{\boldsymbol{k}, n} G(\boldsymbol{k}+\boldsymbol{q}, n) G(\boldsymbol{k}, n)$ is proportional to $z$. By following the same procedure, the threepoint vertex $\Lambda$ is scaled by $z$, and therefore the AL-VC $X^{c}(\mathbf{0}) \sim T U^{4} \sum_{q} \Lambda(\mathbf{0} ; \boldsymbol{q})^{2} \chi^{s}(\boldsymbol{q})^{2}$ is proportional to $z$ under the scaling $T \rightarrow z T$ and $(U, J) \rightarrow(U, J) / z$. Thus, both spin 
and charge irreducible susceptibilities are proportional to $z$, and both $\alpha_{S}$ and $\alpha_{C}$ are unchanged under the rescaling $T \rightarrow z T$ and $(U, J) \rightarrow(U, J) / z$. In other words, the Weiss temperatures $\theta_{S(C)}$ are scaled by $z$. The validity of these scaling relations is confirmed by the numerical study in Fig. 3.

It is possible to obtain $z^{-1}$ by calculating the self-energy $\hat{\Sigma}(k)$ together with $\hat{\chi}^{s, c}(q)$ self-consistently. In this case, fine-tuning of $r$ will be unnecessary since the relation $\alpha_{S, C}<1$ is assured if $\hat{\Sigma}(k)$ and $\hat{\chi}^{s, c}(q)$ are calculated selfconsistently in two-dimensional systems (Mermin-Wagner theorem) [53]. This is an important future issue.

\section{DISCUSSIONS}

\section{A. Spin fluctuation strength and $k$-dependent orbital polarization below $\boldsymbol{T}_{\text {str }}$}

Here, we study the electronic states in the FO order $n_{x z} \neq n_{y z} \quad$ established below the structure transition temperature $T_{\text {str }}$, at which the shear modulus $C_{66}$ reaches zero. According to the linear-response theory, $C_{66} \propto$ $1-g \chi_{x^{2}-y^{2}}^{c}(\mathbf{0})$, where $\chi_{x^{2}-y^{2}}^{c}(\mathbf{0}) \propto\left(T-\theta_{C}\right)^{-1}$ is the electronic orbital susceptibility given by the SC-VC theory, and $g$ is the phonon-mediated Jahn-Teller energy [54]. Therefore, $C_{66} \propto\left(T-T_{\text {str }}\right) /\left(T-\theta_{C}\right)$, and $T_{\text {str }}=\theta_{C}+g$ is slightly higher than $\theta_{C}$ because of the weak electronphonon coupling $(g \approx 10 \sim 50 \mathrm{~K})[2,17,18]$.

Figure 4(a) shows the $T$ dependence of $S_{S}$ given by the RPA for $\mathrm{LaFeAsO}$ and FeSe for $z=1$. Here, we introduce the orbital polarization $-\Delta E / 2(\Delta E / 2)$ for the $d_{x z(y z)}$ level. We put $S_{S}=20$ (5) for LaFeAsO (FeSe) at $T_{\text {str }}=50 \mathrm{meV}$, and we assume a mean-field-type $T$ dependence; $\Delta E=$ $\Delta E_{0} \tanh \left(1.74 \sqrt{T_{\text {str }} / T-1}\right)$ with $\Delta E_{0}=80 \mathrm{meV}$. (For $z^{-1}=4$, the renormalized orbital polarization $z \Delta E_{0}$ is just $20 \mathrm{meV}$.) In both $\mathrm{LaFeAsO}$ and $\mathrm{FeSe}, S_{S}$ are enhanced by $\Delta E$ since $\alpha_{S}$ increases linearly with $\Delta E$ at $\boldsymbol{q}=(\pi, 0)$ as discussed in Ref. [54]. In $\mathrm{LaFeAsO}$, the magnetic-order temperature $T_{\text {mag }}$ increases from $\theta_{S}$ to just below $T_{\text {str }}$ since $S_{S}$ is already large at $T_{\text {str }}$. In contrast, in FeSe, the enhancement of $\chi^{s}(\pi, 0)$ is much more moderate [55].

We also perform the self-consistent analysis of the orbital polarization $\left[\Delta E_{x z}(\boldsymbol{k}), \Delta E_{y z}(\boldsymbol{k})\right]$ and anisotropic $\chi^{s}(\boldsymbol{q})$, which is a natural extension of the SC-VC theory into the orbital ordered state [56]. The obtained $S_{S}$ and $\boldsymbol{k}$ dependent orbital polarizations are shown in Figs. 4(a) (inset) and 4(b), respectively. The parameters are $r=0.256$ and $1 / z_{4}=1.6$. The difference $\Delta n=n_{x z}-n_{y z}$ is $0.2 \%$. The hole pocket around the $\Gamma$ point becomes ellipsoidal along the $k_{y}$ axis because of the "sign-reversing orbital polarization," in which $\Delta E_{x z}(0, k)-\Delta E_{y z}(k, 0)$ shows the sign reversal as shown in Fig. 4(c). Because of this sign reversal, $S_{S}$ in the inset of Fig. 4(a) tends to saturate below $40 \mathrm{meV}$ [33]. Also, two Dirac-cone FSs appear around the $X$ point when $\Delta E_{y z}(\pi, 0)>50 \mathrm{meV}$. These results are
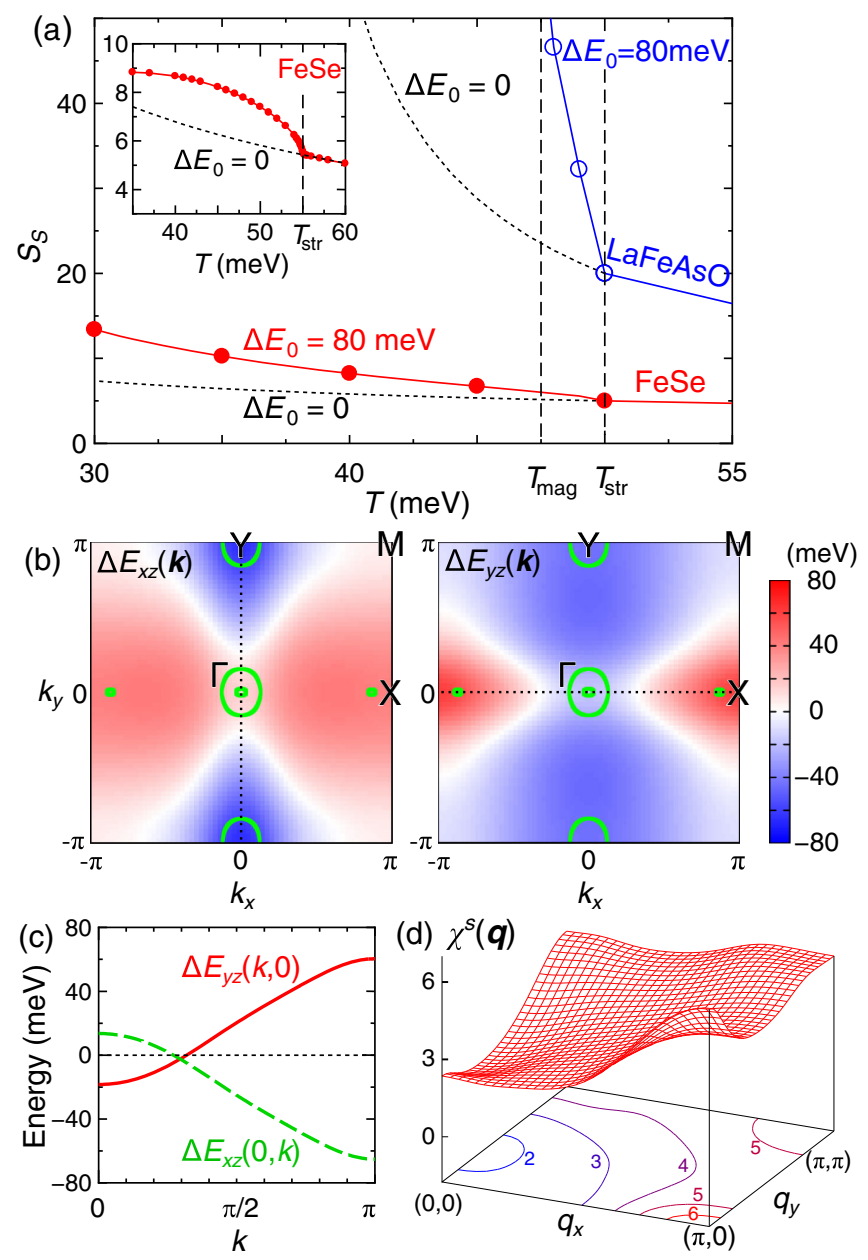

FIG. 4. (a) $T$ dependence of $S_{S}$ for both LaFeAsO and FeSe models $(z=1)$. The FO order is introduced below $T_{\text {str }}=50 \mathrm{meV}$. Inset: $T$ dependence of $S_{S}$ for the FeSe model obtained by calculating the $\boldsymbol{k}$-dependent orbital polarization and $\chi^{s}(\boldsymbol{q})$ self-consistently. $S_{S}$ tends to saturate below $40 \mathrm{meV}$ because of the sign-reversing orbital polarization. (b) Selfconsistent solution of the orbital polarization $\left[\Delta E_{x z}(\boldsymbol{k})\right.$, $\left.\Delta E_{y z}(\boldsymbol{k})\right]$ in the orbital ordered state in the FeSe model at $T=50 \mathrm{meV}$. The shape of the $C_{2}$-symmetric FSs in (b) is consistent with the experimental reports [27-33]. We also show (c) the $\Delta E_{x z(y z)}(\boldsymbol{k})$ along the $k_{y(x)}$ axis and (d) the $C_{2}$-symmetric $\chi^{s}(\boldsymbol{q})$ in the orbital-ordered state.

essentially consistent with the recent ARPES studies reported in Refs. [27-33]. The obtained orbital-polarization $\left[\Delta E_{x z}(\boldsymbol{k}), \Delta E_{y z}(\boldsymbol{k})\right]$ belongs to the $B_{1 g}$ representation, and therefore it is consistent with the " $d$-wave orbital order" discovered in Ref. [31]. The $d$-wave orbital order is theoretically obtained by the mean-field approximation by introducing a phenomenological long-range interaction [57], whose microscopic origin might be the AL-VC studied in this paper.

In the present FeSe model with $z_{4}^{-1}=3, \chi^{s}(\boldsymbol{q}, 0)$ has a maximum at $\boldsymbol{q}=(\pi, 0),(0, \pi)$ without orbital order at $T=50 \mathrm{meV}$, as shown in Fig. 3(a). In the orbital ordered 
state, $\chi^{s}(\boldsymbol{q}, 0)$ at $\boldsymbol{q}=(\pi, 0)$ increases as shown in Fig. 4(d). These results are consistent with the neutron scattering study for FeSe for both $T>T_{\text {str }}$ and $T<T_{\text {str }}$ [4-7]. Essentially similar results are obtained for $z_{4}^{-1}>1.5$ at $T=50 \mathrm{meV}$. We verified that $\chi^{s}(\boldsymbol{q}, 0)$ has a clear maximum peak at $\boldsymbol{q}=(\pi, 0)$ even for $z_{4}^{-1}=1.1$ below $T=$ $10 \mathrm{meV}$ using $128 \times 128 \boldsymbol{k}$ meshes. Experimentally, $z_{2,3} / z_{4}$ is about 3 [27], and the relation $z_{2,3} / z_{4} \sim 1.3$ is predicted by the dynamical-mean-field-theory in Ref. [44].

According to Ref. [4], $\chi^{s}(\boldsymbol{q}, \omega)$ shows the broad maximum at $\boldsymbol{q}=(\pi, 0)$ at low energies $(\omega \lesssim 10 \mathrm{meV})$, and its strength is almost independent for $T>T_{\text {str }}$. The magnitude of the low-energy spin susceptibility in FeSe is 1 order of magnitude smaller than that in $\mathrm{BaFe}_{2} \mathrm{As}_{2}$ [58], whereas its magnitude would be comparable to that in LiFeAs [59]. This experimental report in FeSe will be consistent with the present theoretical result with the moderate $S_{S} \sim 10$ in Figs. 3(c) and 3(d). Note that the experimental dispersion relation in $\chi^{s}(\boldsymbol{q}, \omega)$ for $\omega \lesssim 100 \mathrm{meV}$ is qualitatively understood based on the present FeSe model by considering the band-renormalization factor [7].

\section{B. The ratio $\theta_{S} / \theta_{C}$ for $\mathrm{FeSe}, \mathrm{NaFeAs}, \mathrm{BaFe}_{2} \mathrm{As}_{2}$, and $\mathrm{LaFeAsO}$ as functions of $\overline{\boldsymbol{J}} / \overline{\boldsymbol{U}}$}

In Fig. 5(a), we summarize the ratio $\theta_{S} / \theta_{C}$ obtained in FeSe, $\mathrm{NaFeAs}, \mathrm{BaFe}_{2} \mathrm{As}_{2}$, and $\mathrm{LaFeAsO}$ as a function of $\bar{J} / \bar{U}$. Numerical studies for $\mathrm{NaFeAs}$ and $\mathrm{BaFe}_{2} \mathrm{As}_{2}$ are presented in Appendix C. In $\mathrm{NaFeAs}$ and $\mathrm{FeSe}$, in which $\bar{J} / \bar{U}$ is smaller, the obtained $\theta_{S} / \theta_{C}$ decreases to 0.4 and -0.4 , respectively. In Fig. 5(a), experimental values of $T_{\text {mag }} / T_{\text {str }}$ and $\theta_{\mathrm{NMR}} / T_{\text {str }}$ are also shown, where $\theta_{\mathrm{NMR}}$ is the Weiss temperature of $1 / T_{1} T$ above $T_{\text {str. }}$. Since $T_{\text {str }}=$ $\theta_{C}+g \quad(g \approx 10 \sim 50 \mathrm{~K})$ and $\theta_{\mathrm{NMR}}=\theta_{S}$, the relation $\theta_{\mathrm{NMR}} / T_{\text {str }} \lesssim \theta_{S} / \theta_{C}$ is expected theoretically. In addition, the relation $T_{\mathrm{mag}} / T_{\mathrm{str}} \gtrsim \theta_{S} / \theta_{C}$ is expected since $T_{\mathrm{mag}}$ is substantially higher than $\theta_{S}$ in the FO ordered state. These two theoretically predicted relations are verified in Fig. 5(a). Thus, the ratio $\theta_{S} / \theta_{C}$ is well scaled by the parameter $\bar{J} / \bar{U}$, consistent with the discussion in Sec. IV.

Figure 5(b) shows the critical value of the spin Stoner factor for $\alpha_{C} \approx 1$ in each model, $\alpha_{S}^{\text {cr }}$. It is found that $\alpha_{S}^{\text {cr }}$ increases with $\bar{J} / \bar{U}$ qualitatively. In addition, we plot $\alpha_{S}^{\text {cr }}$ for the FeSe (LaFeAsO) TB model with different Coulomb interactions: $H_{\mathrm{FeSe}(\mathrm{LaFeAsO})}^{0}+r H_{\mathrm{M}}^{U}$. In both FeSe and $\mathrm{LaFeAsO}$ TB models, $\alpha_{S}^{\text {cr }}$ monotonically increases with $\bar{J} / \bar{U}$, whereas $\alpha_{S}^{\text {cr }}$ is clearly small for the FeSe TB model. There are two reasons why $\alpha_{S}^{\text {cr }}$ is smaller for the FeSe band structure. One reason is the absence of the $d_{x y}$-orbital h-FS in FeSe: As we discussed in Sec. IV, the $d_{x y}$-orbital spin fluctuations are unnecessary for the development of $\chi_{x^{2}-y^{2}}^{c}(\mathbf{0})$ because of the AL-VC. Another reason is the smallness of the FSs in FeSe: We found that, numerically, $\alpha_{S}^{\text {cr }}$ decreases when the size of the FS is smaller since the

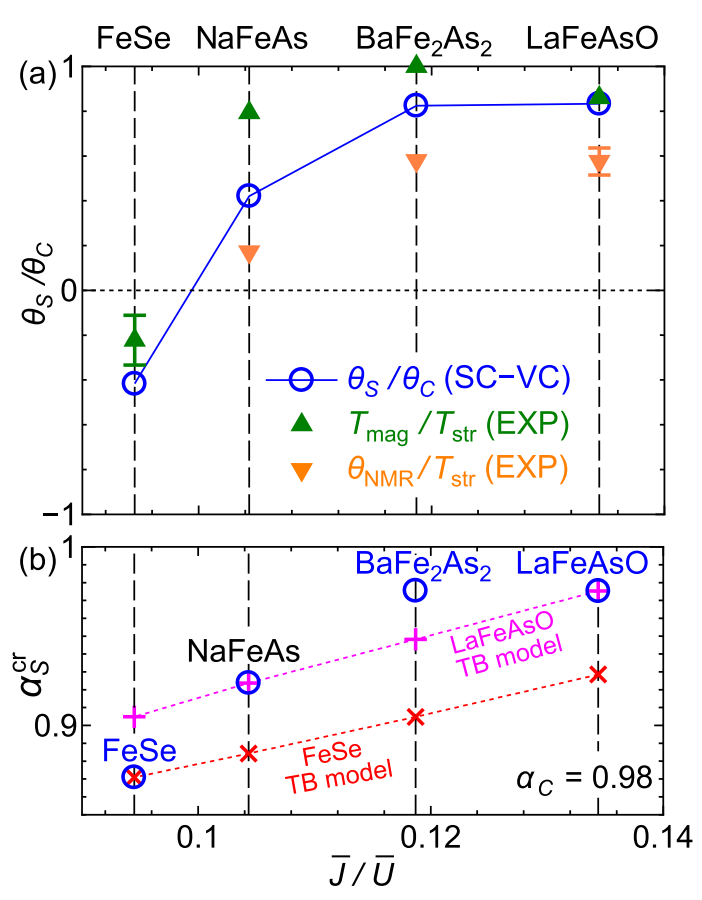

FIG. 5. (a) $\theta_{S} / \theta_{C}$ obtained for four theoretical models. Experimental values of $T_{\text {mag }} / T_{\text {str }}$ and $\theta_{\mathrm{NMR}} / T_{\text {str }}$ are also shown. $\theta_{\mathrm{NMR}}$ is the Weiss temperature of $1 / T_{1} T$ obtained by the CurieWeiss fitting for $T>T_{\text {str }}$ in $\mathrm{LaFeAsO}$ [60], $\mathrm{BaFe}_{2} \mathrm{As}_{2}$ [61], and $\mathrm{NaFeAs}$ [62]. In $\mathrm{LaFeAsO}$, we derived $\theta_{\mathrm{NMR}} \approx 95 \mathrm{~K}$ from the Curie-Weiss fitting of $1 / T_{1} T$ above $T_{\text {str }}$. In FeSe, we derived $T_{\text {mag }}=-10 \sim-30 \mathrm{~K}$ from the Curie-Weiss fitting of $1 / T_{1} T$ below $T_{\text {str }}$ [2]. Thus, theoretically expected relationships $\theta_{\mathrm{NMR}} / T_{\text {str }} \lesssim \theta_{S} / \theta_{C}$ and $T_{\text {mag }} / T_{\text {str }} \gtrsim \theta_{S} / \theta_{C}$ are verified. (b) $\alpha_{S}^{\text {cr }}$ obtained for four theoretical models at $\alpha_{C}=0.98$. We also plot $\alpha_{S}^{\text {cr }}$ for the hybrid models: $H_{\mathrm{LaFeAsO}}^{0}+r H_{\mathrm{M}}^{U}(\mathrm{LaFeAsO}$ TB model $)$ and $H_{\mathrm{FeSe}}^{0}+r H_{\mathrm{M}}^{U}(\mathrm{FeSe} \mathrm{TB}$ model $)$.

three-point vertex $\Lambda_{m} \equiv \delta \chi_{m}^{0}(\boldsymbol{q}) / \delta \Delta E_{m}$, which is odd with respect to $G$, increases in magnitude when the particle-hole asymmetry is large: In fact, we analyzed the undoped $\mathrm{LaFeAsO}$ model with tiny FS pockets by introducing the positive (negative) potential around the electron (hole) FS and verified that the orbital order is realized by small $\alpha_{S}$. Recently, the advantage of the small FSs for the nematicity has been stressed by the renormalization group study in Ref. [63].

\section{Summary}

The emergence of the electronic nematic order has attracted increasing attention as a fundamental phenomenon in strongly correlated metals. In this paper, we studied the origin of the nematicity in Fe-based superconductors, by paying special attention to the nonmagnetic nematic order in FeSe. By applying the orbital + spin fluctuation theory to the first-principles $d-p$ Hubbard models, we succeeded in explaining the rich variety of the phase diagrams in Fe-based superconductors, such as the nonmagnetic or magnetic nematic order in FeSe or LaFeAsO. 
The key model parameter to realize the rich phase diagram is $J / U$ : the ratio between the Hund's and Coulomb interactions. In addition, the ratio $\theta_{S} / \theta_{C}$ tends to decrease as the size of the FS shrinks, as discussed in Sec. VIB.

In both $\mathrm{FeSe}$ and $\mathrm{LaFeAsO}$, strong orbital susceptibility $\chi_{x^{2}-y^{2}}^{c}(\mathbf{0}) \propto\left(T-\theta_{C}\right)^{-1}$ with positive $\theta_{C}$ is realized by the strong orbital-spin interplay due to the strong-coupling effect, called the Aslamazov-Larkin vertex correction in field theory. In the FeSe model, ferro-orbital order is established even when the spin Weiss temperature $\theta_{S}$ is negative, as shown in Fig. 3, since the three-point vertex (the coupling between two magnons and one orbiton) increases at low temperatures as $\Lambda \propto T^{-0.5}$. In contrast, the spin-nematic susceptibility driven by the spin susceptibility should be $T$ independent if $\theta_{S}<0$, as discussed in Ref. [7]. Therefore, we conclude that the nematicity in FeSe originates from the orbital order and fluctuations.

The nematic orbital fluctuations might play important roles in the pairing mechanism in Fe-based superconductors [64]. In FeSe, $T_{\mathrm{c}}$ increases from $9 \mathrm{~K}$ to $40 \mathrm{~K}$ under pressure, accompanied by the enhancement of spin fluctuations [1]. At the same time, the system approaches the orbital critical point since $T_{\text {str }}$ decreases to zero under pressure. These facts indicate the important role of the spin + orbital fluctuations in FeSe.

\section{ACKNOWLEDGMENTS}

We are grateful to A. Chubukov, P. J. Hirschfeld, R. Fernandes, J. Schmalian, Y. Matsuda, T. Shibauchi, and T. Shimojima for useful discussions. This study has been supported by Grants-in-Aid for Scientific Research from Ministry of Education, Culture, Sports, Science, and Technology of Japan.

\section{APPENDIX A: EIGHT-ORBITAL MODELS FOR FeSe AND LaFeAsO}

Here, we introduce the eight-orbital $d$ - $p$ models for FeSe and $\mathrm{LaFeAsO}$ analyzed in the main text. We first derived the first-principles tight-binding models using the WIEN2k and WANNIER90 codes. Crystal structure parameters of $\mathrm{FeSe}$ and $\mathrm{LaFeAsO}$ are given in Refs. [65] and [66], respectively. The obtained band structure and FSs in the LaFeAsO model are shown in Fig. 1 in the main text. In deriving the FeSe model, we introduce the $\boldsymbol{k}$-dependent shifts for orbital $l, \delta E_{l}$, in order to obtain the experimentally observed FSs. In FeSe, we introduce the intra-orbital hopping parameters into $H_{\mathrm{FeSe}}^{0}$ in order to shift the $d_{x y^{-}}$ orbital band $\left(d_{x z / y z}\right.$-orbital band) at $(\Gamma, \mathrm{M}, \mathrm{X})$ points by $(0,-0.25,+0.24)[(-0.24,0,+0.12)]$ in units of eV. Such level shifts are introduced by the additional intra-orbital hopping integrals: $\delta t^{\text {on-site }}=\delta E_{\Gamma} / 4+\delta E_{M} / 4+\delta E_{X} / 2$, $\delta t^{\mathrm{nn}}=\delta E_{\Gamma} / 8-\delta E_{M} / 8$, and $\delta t^{\mathrm{nnn}}=\delta E_{\Gamma} / 16+\delta E_{M} / 16-$ $\delta E_{X} / 8$. The band structure and FSs in the FeSe model are shown in Fig. 1 in the main text.
We also explain the orbital-dependent Coulomb interaction. The bare Coulomb interaction for the spin channel in the main text is

$$
\left(\hat{\Gamma}^{\mathrm{s}}\right)_{l_{1} l_{2}, l_{3} l_{4}}= \begin{cases}U_{l_{1}, l_{1}} & l_{1}=l_{2}=l_{3}=l_{4} \\ U_{l_{1}, l_{2}}^{\prime} & l_{1}=l_{3} \neq l_{2}=l_{4} \\ J_{l_{1}, l_{3}} & l_{1}=l_{2} \neq l_{3}=l_{4} \\ J_{l_{1}, l_{2}} & l_{1}=l_{4} \neq l_{2}=l_{3} \\ 0 & \text { otherwise. }\end{cases}
$$

Also, the bare Coulomb interaction for the charge channel is

$$
\left(\hat{\Gamma}^{\mathrm{c}}\right)_{l_{1} l_{2}, l_{3} l_{4}}= \begin{cases}-U_{l_{1}, l_{1}} & l_{1}=l_{2}=l_{3}=l_{4} \\ U_{l_{1}, l_{2}}^{\prime}-2 J_{l_{1}, l_{2}} & l_{1}=l_{3} \neq l_{2}=l_{4} \\ -2 U_{l_{1}, l_{3}}^{\prime}+J_{l_{1}, l_{3}} & l_{1}=l_{2} \neq l_{3}=l_{4} \\ -J_{l_{1}, l_{2}} & l_{1}=l_{4} \neq l_{2}=l_{3} \\ 0 & \text { otherwise. }\end{cases}
$$

Here, $U_{l, l}, U_{l, l^{\prime}}^{\prime}$, and $J_{l, l^{\prime}}$ are the first-principles Coulomb interaction terms given in Ref. [34].

Finally, we perform the band calculations for the orthorhombic phase of $\mathrm{FeSe}$ and $\mathrm{LaFeAsO}$, based on the experimental crystal structures. In both compounds, the obtained band splitting is too small to explain the large orbital polarization (about $60 \mathrm{meV}$ ) observed by ARPES studies. This result means that the orbital order originates from the electron-electron correlation, which is not included in the band calculation.

Figure 6(a) is the nonmagnetic band structure in the orthorhombic $\mathrm{LaFeAsO}$ obtained by the wIEN2k software. The spin-orbit interaction is not taken into account. The crystal structure parameters in the orthorhombic phase are given in Ref. [66]. The orthorhombic structure deformation $(a-b) /(a+b)$ is $0.3 \%$. Because of the electron-phonon interaction, the fourfold symmetry of the band structure is slightly violated: The splitting between the $d_{x z}$ and $d_{y z}$ bands, $\Delta E^{\text {band }} \equiv E_{y z}-E_{x z}$, is $16 \mathrm{meV}$ at the $X$ point, and $\Delta E^{\text {band }}=2 \mathrm{meV}$ at the $\Gamma$ point.

Figure 6(b) is the band structure in the orthorhombic FeSe. In the orthorhombic phase, the nearest Fe-Fe length is $a=2.6716 \AA$ and $b=2.6610 \AA$, so $(a-b) /(a+b)$ is $0.2 \%$ [65]. Here, the $\boldsymbol{k}$-dependent orbital shift to fit the ARPES band structure introduced above is not taken into account. In FeSe, $\Delta E^{\text {band }}=14 \mathrm{meV}$ at the $X$ point, and $\Delta E^{\text {band }}=3 \mathrm{meV}$ at the $\Gamma$ point. Thus, the sign-reversing orbital splitting observed in Ref. [33] cannot be explained by the band calculation.

The splitting is reduced by the renormalization factor $z$ due to the self-energy. Since $z \sim 1 / 3$ in FeSe and $\mathrm{LaFeAsO}$, the renormalized splitting at the $X$ point is $z \Delta E^{\text {band }} \sim 5 \mathrm{meV}$, which is 1 order of magnitude smaller 

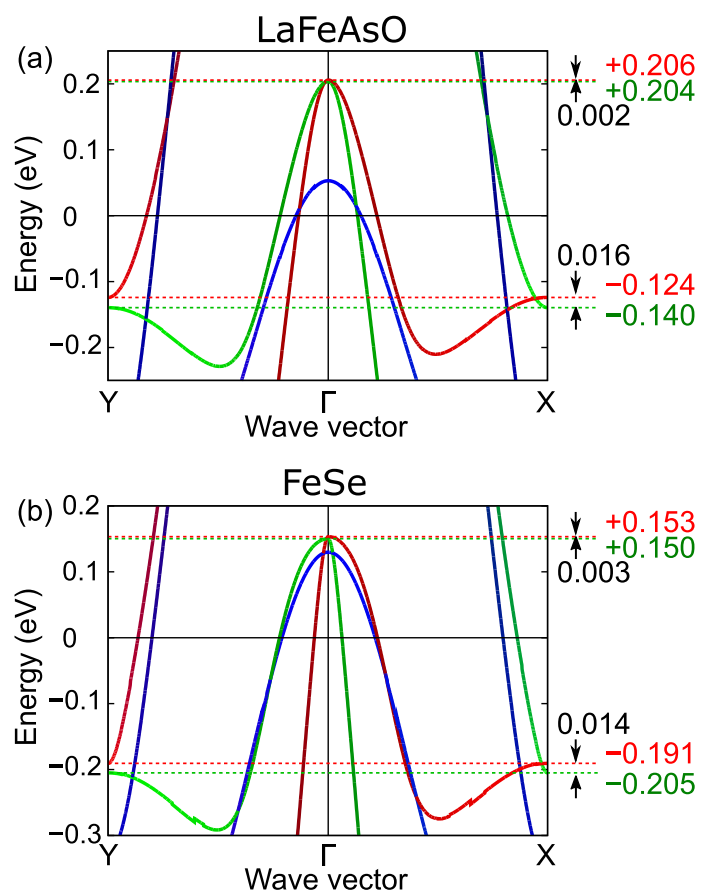

FIG. 6. Band structure of (a) LaFeAsO and (b) FeSe in the experimental orthorhombic crystal structures obtained by the WIEN2k software.

than the experimental orbital splitting. Therefore, it is confirmed that the origin of the electronic nematic state in Fe-based superconductors is the electron-electron correlation.

\section{APPENDIX B: SMALLNESS OF THE VC FOR THE SPIN SUSCEPTIBILITY}

In the original SC-VC theory, the spin and charge susceptibilities are calculated self-consistently by including the MT-VC and AL-VC for the spin and charge susceptibilities $[16,49]$. The strong orbital fluctuations are induced by the charge-channel AL-VC in Fe-based SCs, Ru oxides and cuprate SCs $[16,37,50]$. In the main text, we studied the eight-orbital $d$ - $p$ Hubbard models based on the SC-VC theory by taking the charge-channel AL-VC into account self-consistently. The obtained $\chi^{s}(\boldsymbol{q})$ is equivalent to the RPA since the spin-channel VCs are dropped. It is easy to verify that the charge- and spin-channel MT-VCs are negligible in the present model. However, the smallness of the spin-channel AL-VC is verified only in the twoorbital Hubbard model in Ref. [50].

Here, we study the FeSe model using the SC-VC method by taking the MT-VC and AL-VC for both spin and charge channels in order to confirm the validity of the numerical study in the main text. The charge (spin) susceptibilities are

$$
\hat{\chi}^{c(s)}(\boldsymbol{q})=\hat{\Phi}^{c(s)}(\boldsymbol{q})\left[1-\hat{\Gamma}^{c(s)} \hat{\Phi}^{c(s)}(\boldsymbol{q})\right]^{-1}
$$

where $\hat{\Phi}^{c(s)}(\boldsymbol{q})=\hat{\chi}^{0}(\boldsymbol{q})+\hat{X}^{\mathrm{MT}, c(s)}(\boldsymbol{q})+\hat{X}^{\mathrm{AL}, c(s)}(\boldsymbol{q})$. The spin-channel AL-VC is given as

$$
\begin{aligned}
X_{l, l^{\prime} ; m, m^{\prime}}^{\mathrm{AL}, s}(q)= & \frac{T}{2} \sum_{p} \sum_{a \sim h} \Lambda_{l, l^{\prime} ; a, b ; e, f}(q ; p) \\
& \times\left[\left\{V_{a, b ; c, d}^{c}(p+q) V_{e, f ; g, h}^{s}(-p)\right.\right. \\
& \left.+V_{a, b ; c, d}^{s}(p+q) V_{e, f ; g, h}^{c}(-p)\right\} \\
& \times \Lambda_{m, m^{\prime} ; c, d ; g, h}^{\prime}(q ; p)+2 V_{a, b ; c, d}^{s}(p+q) \\
& \left.\times V_{e, f ; g, h}^{s}(-p) \Lambda_{m, m^{\prime} ; c, d ; g, h}^{\prime \prime}(q ; p)\right],
\end{aligned}
$$

where $\quad \Lambda_{m, m^{\prime} ; c, d ; g, h}^{\prime \prime}(q ; p) \equiv \Lambda_{c, h ; m, g ; d, m^{\prime}}(q ; p)-\Lambda_{g, d ; m, c ; h, m^{\prime}}$ $(q ;-p-q)$. Also, the expressions of the charge- and spin-channel MT-VCs are given in Ref. [49]. The doublecounting second-order terms with respect to $H^{U}$ in $\hat{X}^{\mathrm{MT}, s(c)}+\hat{X}^{\mathrm{AL}, s(c)}$ should be subtracted [49] to obtain reliable results.

Figures 7(a) and 7(b) show the obtained $\chi^{s}(\boldsymbol{q})$ and $\chi_{x^{2}-y^{2}}^{c}(\boldsymbol{q})$, respectively, for $\bar{U}=1.86 \mathrm{eV}(r=0.26)$ at $T=50 \mathrm{meV}$. The Stoner factors are obtained as $\alpha_{S}=$ 0.907 and $\alpha_{C}=0.98$. The obtained VCs $X_{2}^{\mathrm{MT}+\mathrm{AL}, s}(\boldsymbol{q})$ and $X_{2}^{\mathrm{MT}+\mathrm{AL}, c}(\boldsymbol{q})$ for the $d_{x z}$ orbital in the present self-consistent calculation are shown in Figs. 7(c) and 7(d), respectively. Since $\hat{X}^{\mathrm{MT}+\mathrm{AL}, s}(\boldsymbol{q})$ is very small, the obtained charge and spin susceptibilities are very similar to the results in Fig. 3 in the main text. Therefore, the validity of the numerical results in the main text is confirmed by performing the very time-consuming self-consistent calculation with respect to $\hat{X}^{\mathrm{MT}+\mathrm{AL}, s, c}(\boldsymbol{q})$ and $\hat{\chi}^{s, c}(\boldsymbol{q})$.
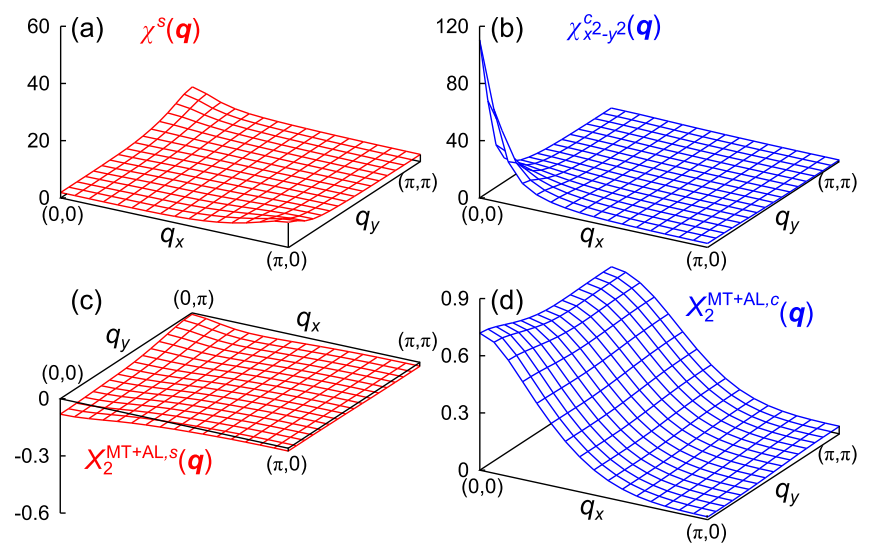

FIG. 7. (a) $\chi^{s}(\boldsymbol{q})$ and (b) $\chi_{x^{2}-y^{2}}^{c}(\boldsymbol{q})$ given by the SC-VC theory, by calculating both spin- and charge-channel VCs selfconsistently. The obtained results are quantitatively equivalent to Fig. 3 in the main text. This fact means that the VC for the spin channel is negligible. (c) $X_{2}^{\mathrm{MT}+\mathrm{AL}, s}(\boldsymbol{q})$ and (d) $X_{2}^{\mathrm{MT}+\mathrm{AL}, c}(\boldsymbol{q})$ obtained by the present self-consistent calculation. 


\section{APPENDIX C: ANALYSIS OF EFFECTIVE MODELS OF $\mathrm{BaFe}_{2} \mathrm{As}_{2}$ AND NaFeAs}

In the main text, we introduced the first-principles models for $\mathrm{LaFeAsO}$ and $\mathrm{FeSe}$ and analyzed these models by using the SC-VC method. Here, we also introduce the effective models for $\mathrm{BaFe}_{2} \mathrm{As}_{2}$ and $\mathrm{NaFeAs}$ and analyze them using the SC-VC method.

In both $\mathrm{BaFe}_{2} \mathrm{As}_{2}$ and $\mathrm{NaFeAs}$, the FSs have relatively large three-dimensional character. In addition, the unfolding of the band structure in $\mathrm{BaFe}_{2} \mathrm{As}_{2}$ cannot be performed exactly because of its body-centered tetragonal crystal structure. Here, we introduce a simple effective $\mathrm{BaFe}_{2} \mathrm{As}_{2} \mathrm{~TB}$ model $H_{\mathrm{BaFe}_{2} \mathrm{As}_{2}}^{0}$ by magnifying the size of
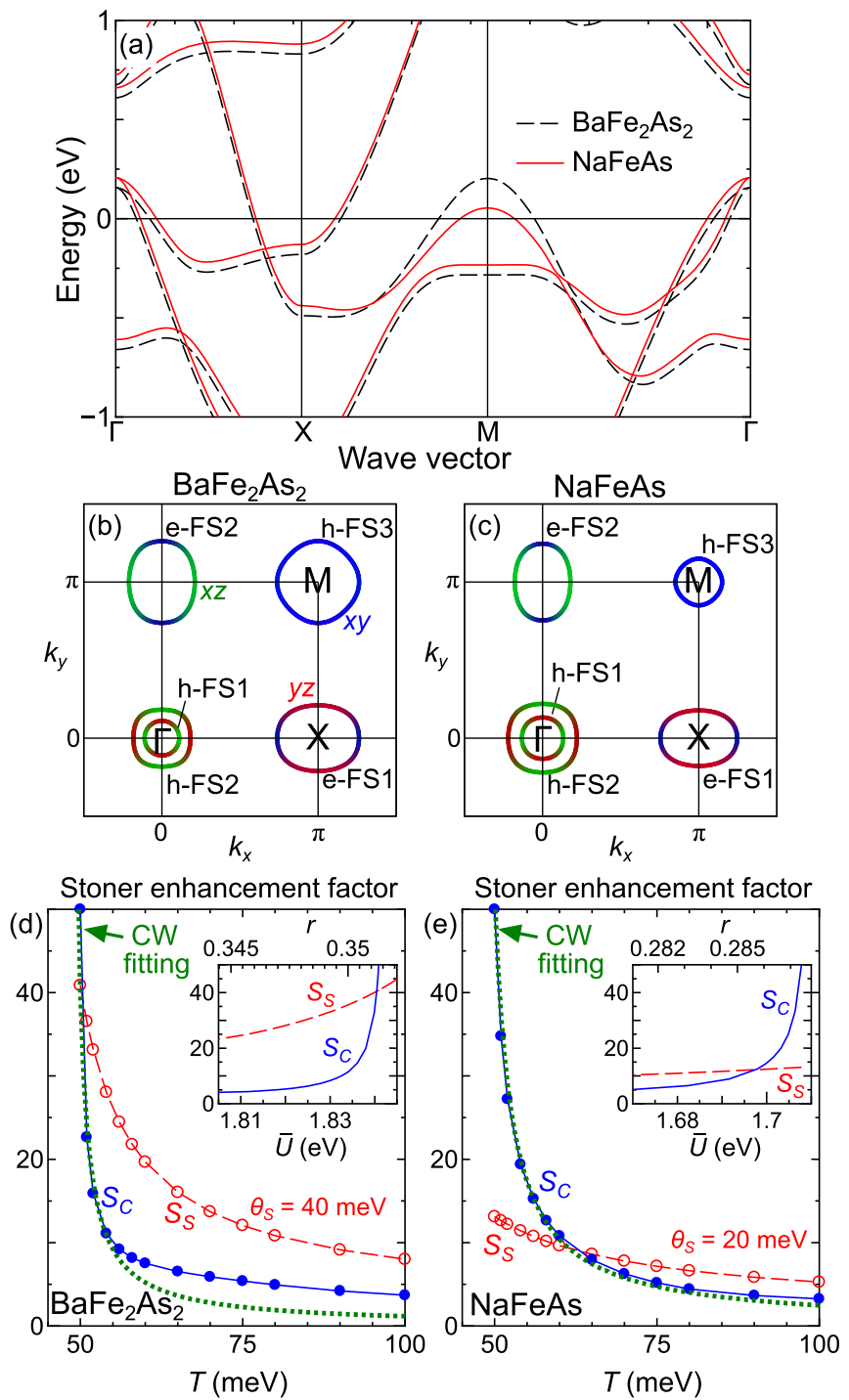

FIG. 8. (a) Band structures of $H_{\mathrm{BaFe}_{2} \mathrm{As}_{2}}^{0}$ and $H_{\mathrm{NaFeAs}}^{0}$. (b) FSs of $H_{\mathrm{BaFe}_{2} \mathrm{As}_{2}}^{0}$ and (c) FSs of $H_{\mathrm{NaFeAs}}^{0}$. (d) $T$ dependence of the spin (charge) Stoner enhancement factors $S_{S(C)}$ obtained in $H_{\mathrm{BaFe}_{2} \mathrm{As}_{2}}$. (Inset) $\bar{U}$ dependence of the Stoner enhancement factors. (e) Spin and charge Stoner enhancement factors in $H_{\mathrm{NaFeAs}}$. the $d_{x y}$-orbital hole-FS around $\boldsymbol{k}=(\pi, \pi)$ in the LaFeAsO unfolded model in order to reproduce the ARPES band structure in Ba122 compounds. Here, we shifted the $d_{x y^{-}}$ orbital band at the $\mathrm{M}$ point by $+0.20 \mathrm{eV}$. As for $\mathrm{NaFeAs}$, we just use $H_{\mathrm{LaFeAsO}}^{0}$ as an effective NaFeAs TB model, e.g., $H_{\mathrm{NaFeAs}}^{0}=H_{\mathrm{LaFeAsO}}^{0}$, considering that the FSs in $\mathrm{NaFeAs}$ in the $k_{z}=0$ plane are similar to the FSs in LaFeAsO. We use $H_{\mathrm{NaFeAs}}^{U}$ in place of $H_{\mathrm{LiFeAs}}^{U}$ given in Ref. [34].

The band structures and the FSs of the effective TB models for $\mathrm{BaFe}_{2} \mathrm{As}_{2}$ and $\mathrm{NaFeAs}$ are shown in Figs. 8(a)-8(c). Here, we perform the SC-VC analysis for the models $H_{\mathrm{M}}=H_{\mathrm{M}}^{0}+r H_{\mathrm{M}}^{U} \quad\left(\mathrm{M}=\mathrm{BaFe}_{2} \mathrm{As}_{2}\right.$, $\mathrm{NaFeAs})$, where $r(<1)$ is the reduction parameter. We choose the parameter $r$ to satisfy the charge Stoner factor, $\alpha_{C}=0.98$. The obtained $T$ dependence of the spin and charge Stoner enhancement factors, $S_{S} \equiv\left(1-\alpha_{S}\right)^{-1}$ and $S_{C} \equiv\left(1-\alpha_{C}\right)^{-1}$, respectively, are shown in Figs. 8(d) and $8(\mathrm{e})$. As for $\mathrm{BaFe}_{2} \mathrm{As}_{2}$, both spin and orbital fluctuations strongly develop at $T \sim 50 \mathrm{meV}$ in the case of $r=0.36$. This result is consistent with the experimental relation $T_{\text {mag }} \approx T_{\text {str }}$ in $\mathrm{BaFe}_{2} \mathrm{As}_{2}$. As for $\mathrm{NaFeAs}$, only orbital fluctuations strongly develop, whereas spin fluctuations remain moderate at $T \sim 50 \mathrm{meV}$ in the case of $r=0.287$. This result is consistent with experimental results in $\mathrm{NaFeAs}$ [62], in which $T_{\mathrm{mag}}(=40 \mathrm{~K})$ is more than 10 Kelvin smaller than $T_{\text {str }}(=53 \mathrm{~K})$. Thus, normalstate phase diagrams in $\mathrm{BaFe}_{2} \mathrm{As}_{2}$ and $\mathrm{NaFeAs}$ are well explained by analyzing their effective Hamiltonians using the SC-VC method.

\section{APPENDIX D: WHY ARE STRONG ORBITAL FLUCTUATIONS INDUCED BY TINY SPIN FLUCTUATIONS IN FeSe?}

In the main text, we studied the first-principles $d-p$ Hubbard models for $\mathrm{LaFeAsO}$ and $\mathrm{FeSe}$ by applying the SC-VC theory. In both models, strong spin-fluctuationdriven orbital fluctuations are induced by AL-VC. In FeSe, we found that very small spin susceptibility $\chi_{\max }^{s}$ is sufficient to realize the orbital order, consistent with experimental results.

Here, we discuss why strong orbital fluctuations are induced by tiny spin fluctuations in FeSe. In Figs. 9(a) and 9(b), we show the spin and orbital susceptibilities, $\chi_{\max }^{s} \equiv \chi^{s}(\boldsymbol{Q}) \quad$ and $\quad \chi_{x^{2}-y^{2}}^{c}(\mathbf{0}) \equiv \chi_{2,2 ; 2,2}^{c}(\boldsymbol{q})+\chi_{3,3 ; 3,3}^{c}(\boldsymbol{q})-$ $2 \chi_{2,2 ; 3,3}^{c}(\mathbf{0})$, in the FeSe model and LaFeAsO model obtained by the SC-VC theory. Here, $32 \times 32 \boldsymbol{k}$ meshes and 256 Matsubara frequencies are used. In both models, the charge Stoner factor is $\alpha_{C}=0.98$ at $T=50 \mathrm{meV}$, and the obtained orbital susceptibilities show similar $T$ dependence. We set $\bar{U}=1.76(r=0.25)$ in FeSe and $\bar{U}=1.74$ $(r=0.41)$ in $\mathrm{LaFeAsO}$, as we did in the main text. As for the spin susceptibility, in $\mathrm{LaFeAsO}$, strong spin fluctuations develop at $T=50 \mathrm{meV}\left(\alpha_{S}=0.98\right)$, consistent with 

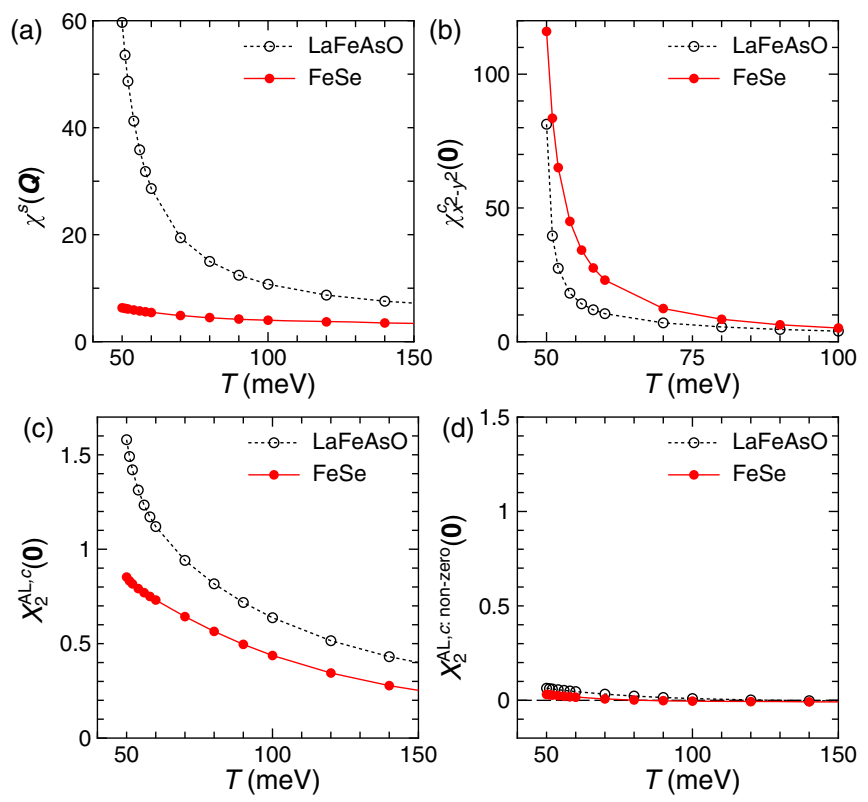

FIG. 9. (a) Spin susceptibility $\chi^{s}(\boldsymbol{Q})$ and (b) orbital susceptibilities $\chi_{x^{2}-y^{2}}^{c}(\mathbf{0})$ for FeSe and $\mathrm{LaFeAsO}$ as functions of $T$. We put $r=0.25$ for FeSe and $r=0.41$ for $\mathrm{LaFeAsO}$. (c) $X_{2}^{\mathrm{AL}, c}(\mathbf{0})$ and (d) $X_{2}^{\mathrm{AL}, c \text { : non-zero }}(\mathbf{0})$ for FeSe and LaFeAsO.

previous theoretical studies [16,37]. In FeSe, in contrast, $\chi_{\max }^{s}$ is almost constant till $T=50 \mathrm{meV}\left(\alpha_{S}=0.87\right)$, consistent with experimental reports in FeSe.

Now, we discuss why the spin fluctuation strength required to realize $\alpha_{C} \approx 1$ is so different from $\mathrm{LaFeAsO}$ to FeSe. One reason is the difference in the ratio $\bar{J} / \bar{U}$ : Figure 9(c) shows the $T$ dependence of the AL-VC on the $d_{x z}$ orbital, $X_{2}^{\mathrm{AL}, c}(\mathbf{0}) \equiv X_{2,2 ; 2,2}^{\mathrm{AL}, c}(\mathbf{0})$, obtained in the $\mathrm{LaFeAsO}$ and FeSe models. In both models, $\alpha_{C}=0.98$ is satisfied at $T=50 \mathrm{meV}$. At $T=50 \mathrm{meV}$, the AL-VC for FeSe is about one-half of that in LaFeAsO. Thus, small AL$\mathrm{VC}$ is enough to induce large orbital fluctuations in $\mathrm{FeSe}$ since the charge Stoner factor is $\alpha_{C} \approx(1-5 \bar{J} / \bar{U}) \bar{U} \Phi_{2}^{c}(\mathbf{0})$.

In Fig. 9(d), we show that $X_{2}^{\mathrm{AL}, \mathrm{c}: \text { nonzero }}(\mathbf{0}) \equiv X_{2}^{\mathrm{AL}, \mathrm{c}}(\mathbf{0})-$ $X_{2}^{\mathrm{AL}, c \text { : zero }}(\mathbf{0})$ is very small for both FeSe and LaFeAsO. Here, "zero" represents the zero-Matsubara term (classical contribution) in Eq. (7) in Sec. II. Thus, nonzero Matsubara terms in the $\mathrm{AL}-\mathrm{VC}$ are negligible in the present calculation (by chance). Note that the $U^{2}$ term in AL-VC gives a negative contribution.

Another reason for the relation $\chi_{\max }^{s}(\mathrm{FeSe}) \ll$ $\chi_{\max }^{s}(\mathrm{LaFeAsO})$ at $\alpha_{C} \approx 1$ is the difference in the orbital dependence of the spin fluctuation strength: The AL-VC for the $x z$ orbital is approximately given as

$$
X_{2}^{\mathrm{AL}, c}(\mathbf{0}) \approx 3 T U^{4} \sum_{\boldsymbol{p}}\left|\Lambda_{2,2 ; 2,2 ; 2,2}(\mathbf{0} ; \boldsymbol{p})\right|^{2} \chi_{2}^{s}(\boldsymbol{p})^{2},
$$

where we dropped the interorbital terms of $\hat{\chi}^{s}$ and $\hat{\Lambda}$, and we leave only the zero-Matsubara term in the Matsubara summation in Eq. (7) in Sec. II. If Eq. (D1) is justified, only the spin fluctuations on the $\left(d_{x z}, d_{y z}\right)$ orbitals are important for the FO fluctuations.

Figure 10(a) shows $\chi_{2}^{s}(\boldsymbol{Q})$ for FeSe and $\mathrm{LaFeAsO}$ for the same model parameters used in Fig. 9. As derived from Fig. 9(a) and Fig. 10(a), the ratio $\chi_{2}^{s}(\boldsymbol{Q}) / \chi^{s}(\boldsymbol{Q})$ is just 0.22 in $\mathrm{LaFeAsO}$, whereas the ratio increases to 0.53 in $\mathrm{FeSe}$ since the relation $\chi_{4}^{s}(\boldsymbol{Q}) \ll \chi_{2}^{s}(\boldsymbol{Q}) \quad\left[\chi_{4}^{s}(\boldsymbol{Q}) \sim \chi_{2}^{s}(\boldsymbol{Q})\right]$ is satisfied in $\mathrm{FeSe}(\mathrm{LaFeAsO})$ because of the absence (presence) of h-FS3. This orbital dependence of the spin fluctuations in $\mathrm{FeSe}$ is favorable for realizing the FO fluctuations.

To understand the model dependence of the AL-VC in more detail, we calculate $C_{2} \equiv \sum_{q} \chi_{2}^{s}(\boldsymbol{q})^{2}$ and show the result in Fig. 10(b): The ratio $C_{2}^{\mathrm{LaFeAsO}} / C_{2}^{\mathrm{FeSe}}$ is just 1.35 since the width of the peak of $\chi_{2}^{s}(\boldsymbol{q})^{2}$ around $\boldsymbol{q}=\boldsymbol{Q}$ is much wider in FeSe. We also examine the square of the threepoint vertex for the $d_{x z}$ orbital $\Lambda_{2} \equiv \Lambda_{2,2 ; 2,2 ; 2,2}(\boldsymbol{q}, \boldsymbol{p})$ at $\boldsymbol{q}=\mathbf{0}$ and $\boldsymbol{p}=\boldsymbol{Q}$ in Fig. 10(c). In both models, the relation $\left|\Lambda_{2}\right|^{2} \propto T^{-a}$ with $a \approx 1$ is satisfied for a wide temperature range: Such strong $T$ dependence of the charge-spin coupling $\Lambda_{2}$ is essential for realizing the orbital fluctuations, so it should be taken into account in the numerical calculation. As a result, we obtain a crude approximation for the $\mathrm{AL}-\mathrm{VC}, \tilde{X}_{2}^{\mathrm{AL}, c} \equiv 3 U^{4}\left|\Lambda_{2}(\mathbf{0} ;(0, \pi))\right|^{2} T C_{2}$, and show the result in Fig. 10(d). This crude approximation qualitatively reproduces the exact numerical results for both $\mathrm{FeSe}$ and $\mathrm{LaFeAsO}$ given in Fig. 9(c).
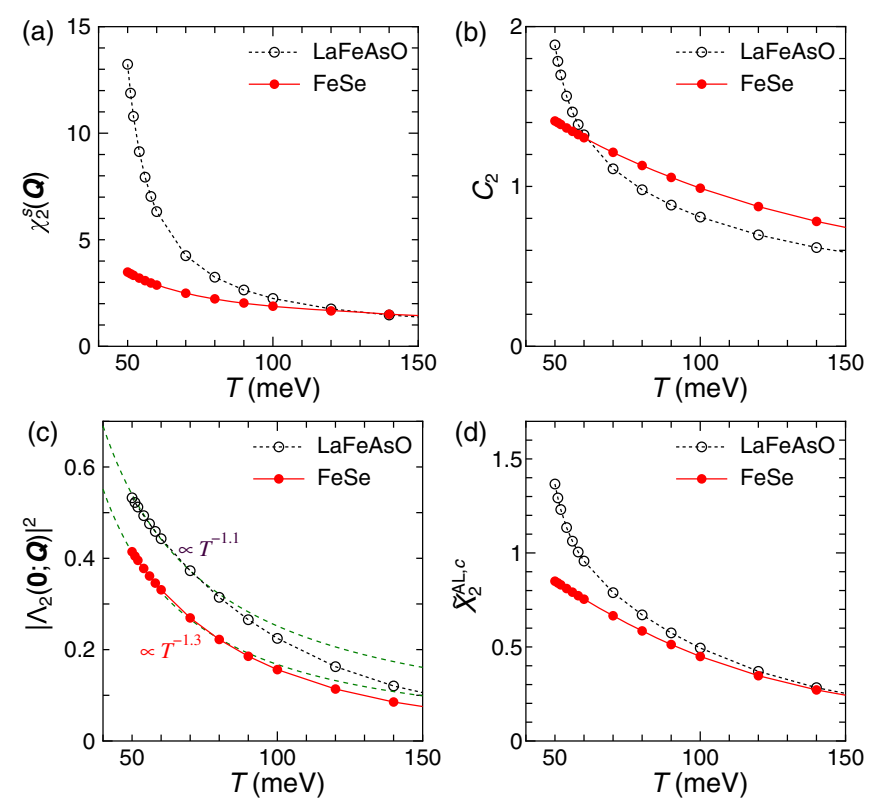

FIG. 10. (a) $\chi_{2}^{s}(\boldsymbol{Q})$, (b) $C_{2} \equiv \sum_{q} \chi_{2}^{s}(\boldsymbol{q})^{2}$, and (c) $\left|\Lambda_{2}\right|^{2}$ as functions of $T$ in FeSe and $\mathrm{LaFeAsO}$. (d) The approximate $\mathrm{AL}-\mathrm{VC}$ for the $d_{x z}$ orbital $\tilde{X}_{2}^{\mathrm{AL}, c} \equiv 3 U^{4}\left|\Lambda_{2}(\mathbf{0} ;(0, \pi))\right|^{2} T C_{2}$. In both FeSe and $\mathrm{LaFeAsO}$, the obtained $\tilde{X}_{2}^{\mathrm{AL}, c}$ qualitatively agrees with the exact numerical calculations in Fig. 9(c). 
In summary, in both $\mathrm{LaFeAsO}$ and $\mathrm{FeSe}$, strong orbital fluctuations are induced by AL-VC for the $d_{x z(y z)}$ orbital, $X_{2(3)}^{\mathrm{AL}, c}(\mathbf{0})$. In FeSe, very small spin susceptibility $\chi_{\max }^{s}$ is sufficient to realize the spin-fluctuation-driven orbital order because of both the smallness of $\bar{J} / \bar{U}$ and the largeness of $C_{2}$. Strong $T$ dependence of $\Lambda_{2}$ is essential for realizing the orbital fluctuations due to AL-VC.

\section{APPENDIX E: STRONG $T$ DEPENDENCE OF THE THREE-POINT VERTEX}

In this paper, we found that the strong orbital fluctuations in Fe-based superconductors originate from the AL-VC for the orbital susceptibility. The moderate increment of the AL-VC at low temperatures shown in Fig. 9(c) gives rise to the Curie-Weiss behavior of $\chi_{x^{2}-y^{2}}^{c}(\mathbf{0})$. For the increment of the AL-VC, the strong $T$ dependence of the three-point vertex, shown in Fig. 10(c), plays a significant role. Its strong $T$ dependence in Fe-based superconductors has been pointed out in Refs. [20,21,51,52].

Here, we calculate the three-point vertex for $\mathrm{LaFeAsO}$ and $\mathrm{FeSe}$ models for a wide temperature range with high numerical accuracy, using $512 \times 512 \boldsymbol{k}$ meshes and about 2048 Matsubara frequencies. Figure 11 shows the square of the three-point vertex for the $d_{x z}$ orbital $\Lambda_{2}(\mathbf{0} ; \boldsymbol{Q}) \equiv$ $\Lambda_{2,2 ; 2,2 ; 2,2}(\mathbf{0} ; \boldsymbol{Q})$ for $T \geq 10 \mathrm{meV}$. In both $\mathrm{LaFeAsO}$ and FeSe models, the coefficient $a$ of $\left|\Lambda_{2}(\mathbf{0} ; \boldsymbol{Q})\right|^{2} \propto T^{-a}$ depends on the temperature range. In both models, $a \approx 1$ for $T=20 \sim 100 \mathrm{meV}$, so the numerical result in Fig. 10(c) is confirmed by this accurate calculation. When the band renormalization due to $z<1$ is considered, the relation $a \approx 1$ is realized for $T=20 z \sim 100 z[\mathrm{meV}]$.

For $T<20 z[\mathrm{meV}],\left|\Lambda_{2}(\mathbf{0} ; \boldsymbol{Q})\right|^{2}$ saturates since the temperature is smaller than the energy scale of the nesting. For $T>100 z[\mathrm{meV}]$, the relation $a \approx 2$ is realized as discussed in Refs. [51,52]. Note that the chemical potential $\mu$ becomes higher than that of the hole band at the $\Gamma$ point when $T$ is higher than $100 z(300 z)[\mathrm{meV}]$ in the FeSe (LaFeAsO) model.

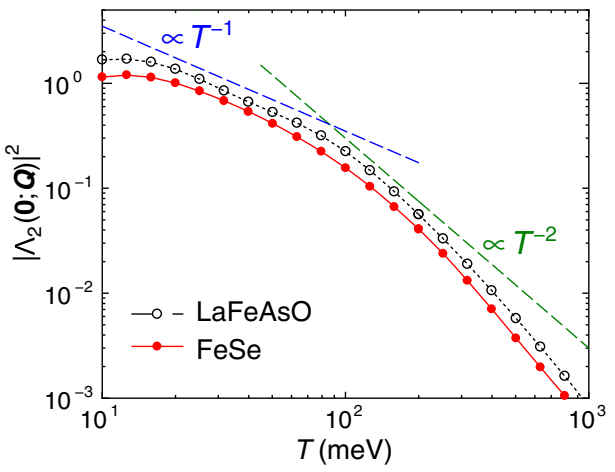

FIG. 11. $T$ dependence of $\left|\Lambda_{2}(\mathbf{0} ; \boldsymbol{Q})\right|^{2}$ in $\mathrm{LaFeAsO}$ and FeSe models. Here, $512 \times 512 \boldsymbol{k}$ meshes are used.
In Ref. [20], we reported the relation $X_{2}^{\mathrm{AL}, c}(\mathbf{0}) / T \propto$ $T^{-0.5}\left(1-\alpha_{S}\right)^{-1}$ based on an approximate calculation for the five-orbital $\mathrm{LaFeAsO} \mathrm{TB}$ model. The factor $T^{-0.5}$ originates from $\left|\Lambda_{2}\right|^{2}$. However, we performed a more careful numerical analysis and found that the approximate relation $\left|\Lambda_{2}\right|^{2} \sim T^{-1}(a \approx 1)$ is realized for $T \sim 50 z[\mathrm{meV}]$ in the five-orbital model.

\section{APPENDIX F: TWO DEFINITIONS OF THE AVERAGED COULOMB AND HUND'S INTERACTIONS}

In the present study, the ratio between the intra-orbital Coulomb interaction and Hund's interaction, $\bar{J} / \bar{U}$, is the essential control parameter. In this paper, we follow the Hubbard-Kanamori definition: $\bar{U} \equiv \frac{1}{5} \sum_{l=1}^{5} U_{l, l}$ and $\bar{J} \equiv \frac{1}{10} \sum_{l>m} J_{l, m}$. This definition is used in Ref. [34]. By using the Slater integrals [67], it is expressed as $\bar{U}=F^{0}+\frac{4}{49}\left(F^{2}+F^{4}\right), \bar{U}^{\prime}=F^{0}-\frac{1}{49}\left(F^{2}+F^{4}\right)$, and $\bar{J}=$ $\frac{5}{98}\left(F^{2}+F^{4}\right)$ [67]. According to the first-principles cRPA method [34], the ratio $\bar{J} / \bar{U}$ is 0.0945 for FeSe, and the relation $\bar{U}^{\prime}=\bar{U}-2 \bar{J}$ is approximately satisfied.

In the first-principles studies, another definition of the averaged interaction is used frequently: $\tilde{U}=F^{0}$ and $\tilde{J}=\frac{1}{14}\left(F^{2}+F^{4}\right)$. This definition is used in Refs. [68,69]. It is equivalent to $\tilde{U}=\frac{1}{25}\left(\sum_{l=1}^{5} U_{l, l}+\sum_{l \neq m} U_{l, m}^{\prime}\right)$ and $\tilde{J}=$ $\tilde{U}-\frac{1}{10} \sum_{l \neq m}\left(U_{l, m}^{\prime}-J_{l, m}\right)[70,71]$.

Therefore, if we assume $\bar{U}^{\prime}=\bar{U}-2 \bar{J}$, which is actually satisfied well in Ref. [34], we obtain the relations $\tilde{U}=$ $\bar{U}-\frac{8}{5} \bar{J}$ and $\tilde{J}=\frac{7}{5} \bar{J}$. Thus, $\tilde{J} / \tilde{U}=0.224$ obtained by the first-principles study for FeSe in Ref. [68] corresponds to $\bar{J} / \bar{U}=0.127$. Also, $\tilde{J} / \tilde{U}=0.294$ for $\mathrm{LaFeAsO}$ obtained in Refs. $[68,69]$ corresponds to $\bar{J} / \bar{U}=0.157$.

As shown in Ref. 5(b), the value of $\alpha_{S}^{\text {cr }}$ remains small (about 0.9) in the FeSe TB model with $\hat{H}_{\mathrm{BaFe}_{2} \mathrm{As}_{2}}^{U}$ $(\bar{J} / \bar{U}=0.12)$ or with $\hat{H}_{\mathrm{LaFeAsO}}^{U}(\bar{J} / \bar{U}=0.134)$. In each case, the obtained $T$ dependence of $S_{S}$ and $S_{C}$ is qualitatively similar to that shown in Fig. 3(c). Therefore, the main results of the present study are unchanged even if $\bar{J} / \bar{U}$ in FeSe is slightly larger than 0.1 .

[1] D. C. Johnston, The Puzzle of High Temperature Superconductivity in Layered Iron Pnictides and Chalcogenides, Adv. Phys. 59, 803 (2010); Y. Mizuguchi and Y. Takano, Review of $\mathrm{Fe}$ Chalcogenides as the Simplest Fe-Based Superconductor, J. Phys. Soc. Jpn. 79, 102001 (2010).

[2] A. E. Böhmer, T. Arai, F. Hardy, T. Hattori, T. Iye, T. Wolf, H. v. Lohneysen, K. Ishida, and C. Meingast, Origin of the Tetragonal-to-Orthorhombic Phase Transition in FeSe: A Combined Thermodynamic and NMR Study of Nematicity, Phys. Rev. Lett. 114, 027001 (2015). 
[3] S.-H. Baek, D. V. Efremov, J. M. Ok, J. S. Kim, J. van den Brink, and B. Büchner, Orbital-Driven Nematicity in FeSe, Nat. Mater. 14, 210 (2015).

[4] M. C. Rahn, R. A. Ewings, S. J. Sedlmaier, S. J. Clarke, and A. T. Boothroyd, Strong $(\pi, 0)$ Spin Fluctuations in $\beta-$ FeSe Observed by Neutron Spectroscopy, Phys. Rev. B 91, 180501(R) (2015).

[5] Q. Wang, Y. Shen, B. Pan, Y. Hao, M. Ma, F. Zhou, P. Steffens, K. Schmalzl, T. R. Forrest, M. Abdel-Hafiez et al., Strong Interplay between Stripe Spin Fluctuations, Nematicity and Superconductivity in FeSe, Nat. Mater. 15, 159 (2016).

[6] Q. Wang, Y. Shen, B. Pan, X. Zhang, K. Ikeuchi, K. Iida, A. D. Christianson, H. C. Walker, D. T. Adroja, M. Abdel-Hafiez et al., Magnetic Ground State of FeSe, arXiv:1511.02485.

[7] S. Shamoto, K. Matsuoka, R. Kajimoto, M. Ishikado, Y. Yamakawa, T. Watashige, S. Kasahara, M. Nakamura, H. Kontani, T. Shibauchi, and Y. Matsuda, Spin Nematic Susceptibility Studied by Inelastic Neutron Scattering in FeSe, arXiv:1511.04267.

[8] R. M. Fernandes, L. H. VanBebber, S. Bhattacharya, P. Chandra, V. Keppens, D. Mandrus, M. A. McGuire, B. C. Sales, A. S. Sefat, and J. Schmalian, Effects of Nematic Fluctuations on the Elastic Properties of Iron Arsenide Superconductors, Phys. Rev. Lett. 105, 157003 (2010).

[9] F. Wang, S. A. Kivelson, and D.-H. Lee, Nematicity and Quantum Paramagnetism in FeSe, Nat. Phys. 11, 959 (2015).

[10] A. V. Chubukov, R. M. Fernandes, and J. Schmalian, Origin of Nematic Order in FeSe, Phys. Rev. B 91, 201105(R) (2015).

[11] J. K. Glasbrenner, I. I. Mazin, H. O. Jeschke, P. J. Hirschfeld, R. M. Fernandes, and R. Valentí, Effect of Magnetic Frustration on Nematicity and Superconductivity in Iron Chalcogenides, Nat. Phys. 11, 953 (2015).

[12] R. Yu and Q. Si, Antiferroquadrupolar and Ising-Nematic Orders of a Frustrated Bilinear-Biquadratic Heisenberg Model and Implications for the Magnetism of FeSe, Phys. Rev. Lett. 115, 116401 (2015).

[13] F. Krüger, S. Kumar, J. Zaanen, and J. van den Brink, Spin-Orbital Frustrations and Anomalous Metallic State in Iron-Pnictide Superconductors, Phys. Rev. B 79, 054504 (2009).

[14] W. Lv, J. Wu, and P. Phillips, Orbital Ordering Induces Structural Phase Transition and the Resistivity Anomaly in Iron Pnictides, Phys. Rev. B 80, 224506 (2009).

[15] C.-C. Lee, W.-G. Yin, and W. Ku, Ferro-Orbital Order and Strong Magnetic Anisotropy in the Parent Compounds of Iron-Pnictide Superconductors, Phys. Rev. Lett. 103, 267001 (2009).

[16] S. Onari and H. Kontani, Self-Consistent Vertex Correction Analysis for Iron-based Superconductors: Mechanism of Coulomb Interaction-Driven Orbital Fluctuations, Phys. Rev. Lett. 109, 137001 (2012).

[17] M. Yoshizawa, D. Kimura, T. Chiba, S. Simayi, Y. Nakanishi, K. Kihou, C.-H. Lee, A. Iyo, H. Eisaki, M. Nakajima, and S. Uchida, Structural Quantum Criticality and Superconductivity in Iron-Based Superconductor $\mathrm{Ba}\left(\mathrm{Fe}_{1-x} \mathrm{Co}_{x}\right)_{2} \mathrm{As}_{2}$, J. Phys. Soc. Jpn. 81, 024604 (2012).
[18] A. E. Böhmer, P. Burger, F. Hardy, T. Wolf, P. Schweiss, R. Fromknecht, M. Reinecker, W. Schranz, and C. Meingast, Nematic Susceptibility of Hole-Doped and Electron-Doped $\mathrm{BaFe}_{2} \mathrm{As}_{2}$ Iron-Based Superconductors from Shear Modulus Measurements, Phys. Rev. Lett. 112, 047001 (2014).

[19] Y. Gallais, R. M. Fernandes, I. Paul, L. Chauvière, Y.-X. Yang, M.-A. Méasson, M. Cazayous, A. Sacuto, D. Colson, and A. Forget, Observation of Incipient Charge Nematicity in $\mathrm{Ba}\left(\mathrm{Fe}_{1-X} \mathrm{Co}_{X}\right)_{2} \mathrm{As}_{2}$, Phys. Rev. Lett. 111, 267001 (2013).

[20] H. Kontani and Y. Yamakawa, Linear Response Theory for Shear Modulus $C_{66}$ and Raman Quadrupole Susceptibility: Evidence for Nematic Orbital Fluctuations in Fe-based Superconductors, Phys. Rev. Lett. 113, 047001 (2014).

[21] M. Khodas and A. Levchenko, Raman Scattering as a Probe of Nematic Correlations, Phys. Rev. B 91, 235119 (2015).

[22] U. Karahasanovic, F. Kretzschmar, T. Böhm, R. Hackl, I. Paul, Y. Gallais, and J. Schmalian, Manifestation of Nematic Degrees of Freedom in the Raman Response Function of Iron Pnictides, Phys. Rev. B 92, 075134 (2015).

[23] J.-H. Chu, H.-H. Kuo, J. G. Analytis, and I. R. Fisher, Divergent Nematic Susceptibility in an Iron Arsenide Superconductor, Science 337, 710 (2012).

[24] H.-H. Kuo, J.-H. Chu, J. C. Palmstrom, S. A. Kivelson, and I. R. Fisher, Ubiquitous Signatures of Nematic Quantum Criticality in Optimally Doped Fe-based Superconductors, arXiv:1503.00402.

[25] C. J. Halboth and W. Metzner, $d$-Wave Superconductivity and Pomeranchuk Instability in the Two-Dimensional Hubbard Model, Phys. Rev. Lett. 85, 5162 (2000); C. Honerkamp, M. Salmhofer, N. Furukawa, and T. M. Rice, Breakdown of the Landau-Fermi Liquid in Two Dimensions Due to Umklapp Scattering, Phys. Rev. B 63, 035109 (2001); H. Yamase and H. Kohno, Instability toward Formation of Quasi-OneDimensional Fermi Surface in Two-Dimensional t-J Model, J. Phys. Soc. Jpn. 69, 2151 (2000).

[26] M. Yi, D. Lu, J.-H. Chu, J. G. Analytis, A. P. Sorini, A. F. Kemper, B. Moritz, S.-K. Mo, R. G. Moore, M. Hashimoto et al., Symmetry-Breaking Orbital Anisotropy Observed for Detwinned $\mathrm{Ba}\left(\mathrm{Fe}_{1-x} \mathrm{Co}_{x}\right)_{2} \mathrm{As}_{2}$ above the Spin Density Wave Transition, Proc. Natl. Acad. Sci. U.S.A. 108, 6878 (2011).

[27] J. Maletz, V. B. Zabolotnyy, D. V. Evtushinsky, S. Thirupathaiah, A. U. B. Wolter, L. Harnagea, A. N. Yaresko, A. N. Vasiliev, D. A. Chareev, A. E. Böhmer et al., Unusual Band Renormalization in the Simplest Iron-Based Superconductor FeSe $\mathrm{F}_{1-x}$, Phys. Rev. B 89, 220506(R) (2014).

[28] K. Nakayama, Y. Miyata, G. N. Phan, T. Sato, Y. Tanabe, T. Urata, K. Tanigaki, and T. Takahashi, Reconstruction of Band Structure Induced by Electronic Nematicity in an FeSe Superconductor, Phys. Rev. Lett. 113, 237001 (2014).

[29] M. D. Watson, T. K. Kim, A. A. Haghighirad, N. R. Davies, A. McCollam, A. Narayanan, S. F. Blake, Y. L. Chen, S. Ghannadzadeh, A. J. Schofield et al., Emergence of the Nematic Electronic State in FeSe, Phys. Rev. B 91, 155106 (2015).

[30] T. Shimojima, Y. Suzuki, T. Sonobe, A. Nakamura, M. Sakano, J. Omachi, K. Yoshioka, M. Kuwata-Gonokami, K. Ono, H. Kumigashira et al., Lifting of $x z / y z$ rbital Degeneracy at the Structural Transition in Detwinned FeSe, Phys. Rev. B 90, 121111(R) (2014). 
[31] P. Zhang, T. Qian, P. Richard, X. P. Wang, H. Miao, B. Q. Lv, B. B. Fu, T. Wolf, C. Meingast, X.X. Wu et al., Observation of Two Distinct $d_{x z} / d_{y z}$ Band Splittings in FeSe, Phys. Rev. B 91, 214503 (2015).

[32] Y. Zhang, M. Yi, Z.-K. Liu, W. Li, J. J. Lee, R. G. Moore, M. Hashimoto, N. Masamichi, H. Eisaki, S.-K. Mo et al., Distinctive Momentum Dependence of the Band Reconstruction in the Nematic State of FeSe Thin Film, arXiv:1503.01556.

[33] Y. Suzuki, T. Shimojima, T. Sonobe, A. Nakamura, M. Sakano, H. Tsuji, J. Omachi, K. Yoshioka, M. KuwataGonokami, T. Watashige et al., Momentum-Dependent Sign-Inversion of Orbital Polarization in Superconducting FeSe, Phys. Rev. B 92, 205117 (2015).

[34] T. Miyake, K. Nakamura, R. Arita, and M. Imada, Comparison of Ab Initio Low-Energy Models for LaFePO, LaFeAsO, $\mathrm{BaFe}_{2} \mathrm{As}_{2}$, LiFeAs, FeSe, and FeTe: Electron Correlation and Covalency, J. Phys. Soc. Jpn. 79, 044705 (2010).

[35] M. Tsuchiizu, Y. Ohno, S. Onari, and H. Kontani, Orbital Nematic Instability in the Two-Orbital Hubbard Model: Renormalization-Group + Constrained RPA Analysis, Phys. Rev. Lett. 111, 057003 (2013).

[36] M. Tsuchiizu, Y. Yamakawa, S. Onari, Y. Ohno, and H. Kontani, Spin-Triplet Superconductivity in $\mathrm{Sr}_{2} \mathrm{RuO}_{4}$ Due to Orbital and Spin Fluctuations: Analyses by Two-Dimensional Renormalization Group Theory and Self-Consistent Vertex-Correction Method, Phys. Rev. B 91, 155103 (2015).

[37] S. Onari, Y. Yamakawa, and H. Kontani, High- $T_{c}$ Superconductivity near the Anion Height Instability in Fe-Based Superconductors: Analysis of $\mathrm{LaFeAsO}_{1-x} \mathrm{H}_{x}$, Phys. Rev. Lett. 112, 187001 (2014).

[38] Y. Yamakawa and H. Kontani, Spin-Fluctuation-Driven Nematic Charge-Density Wave in Cuprate Superconductors: Impact of Aslamazov-Larkin Vertex Corrections, Phys. Rev. Lett. 114, 257001 (2015).

[39] M. Tsuchiizu, Y. Yamakawa, and H. Kontani, p-Orbital Density Wave with d Symmetry in High- $T_{c}$ Cuprate Superconductors, Phys. Rev. B 93, 155148 (2016).

[40] C. Xu, M. Müller, and S. Sachdev, Ising and Spin Orders in the Iron-Based Superconductors, Phys. Rev. B 78, 020501(R) (2008); C. Fang, H. Yao, W.-F. Tsai, J. P. Hu, and S. A. Kivelson, Theory of Electron Nematic Order in LaFeAsO, Phys. Rev. B 77, 224509 (2008); E. Abrahams and Q. Si, Quantum Criticality in the Iron Pnictides and Chalcogenides, J. Phys. Condens. Matter 23, 223201 (2011).

[41] A. V. Chubukov and P. J. Hirschfeld, Iron-Based Superconductors, Seven Years Later, Phys. Today, 68, 46 (2015); H. Hosono and K. Kuroki, Iron-Based Superconductors: Current Status of Materials and Pairing Mechanism, Physica (Amsterdam) 514C, 399 (2015).

[42] T. Terashima, N. Kikugawa, A. Kiswandhi, E.-S. Choi, J. S. Brooks, S. Kasahara, T. Watashige, H. Ikeda, T. Shibauchi, Y. Matsuda et al., Anomalous Fermi Surface in FeSe Seen by Shubnikov-de Haas Oscillation Measurements, Phys. Rev. B 90, 144517 (2014).

[43] A. Audouard, F. Duc, L. Drigo, P. Toulemonde, S. Karlsson, P. Strobel, and A. Sulpice, Quantum Oscillations and Upper
Critical Magnetic Field of the Iron-Based Superconductor FeSe, Europhys. Lett. 109, 27003 (2015).

[44] Z. P. Yin, K. Haule, and G. Kotliar, Kinetic Frustration and the Nature of the Magnetic and Paramagnetic States in Iron Pnictides and Iron Chalcogenides, Nat. Mater. 10, 932 (2011); J. Ferber, K. Foyevtsova, R. Valentí, and H. O. Jeschke, LDA + DMFT Study of the Effects of Correlation in LiFeAs, Phys. Rev. B 85, 094505 (2012); G. Lee, H. S. Ji, Y. Kim, C. Kim, K. Haule, G. Kotliar, B. Lee, S. Khim, K. H. Kim, K. S. Kim, K.-S. Kim, and J. H. Shim, Orbital Selective Fermi Surface Shifts and Mechanism of High $T_{c}$ Superconductivity in Correlated AFeAs $(A=\mathrm{Li}, N a)$, Phys. Rev. Lett. 109, 177001 (2012).

[45] S. Kasahara, T. Watashige, T. Hanaguri, Y. Kohsaka, T. Yamashita, Y. Shimoyama, Y. Mizukami, R. Endo, H. Ikeda, K. Aoyama et al., Field-Induced Superconducting Phase of FeSe in the BCS-BEC Cross-over, Proc. Natl. Acad. Sci. U.S.A. 111, 16309 (2014).

[46] R. Arita, private communication.

[47] K. Suzuki, H. Usui, and K. Kuroki, Possible ThreeDimensional Nodes in the $\mathrm{s} \pm$ Superconducting Gap of $\mathrm{BaFe}_{2}\left(\mathrm{As}_{1-x} \mathrm{P}_{x}\right)_{2}$, J. Phys. Soc. Jpn. 80, 013710 (2011).

[48] T. Misawa and M. Imada, Superconductivity and Its Mechanism in an Ab Initio Model for Electron-Doped LaFeAsO, Nat. Commun. 5, 5738 (2014).

[49] S. Onari and H. Kontani, Iron-Based Superconductivity, edited by P. D. Johnson, G. Xu, and W.-G. Yin (SpringerVerlag, Berlin and Heidelberg, 2015).

[50] Y. Ohno, M. Tsuchiizu, S. Onari, and H. Kontani, SpinFluctuation-Driven Orbital Nematic Order in Ru-Oxides: Self-Consistent Vertex Correction Analysis for Two-Orbital Model, J. Phys. Soc. Jpn. 82, 013707 (2013).

[51] A. Hinojosa, J. Cai, and A. V. Chubukov, Raman Resonance in Iron-Based Superconductors: The Magnetic Scenario, Phys. Rev. B 93, 075106 (2016).

[52] I. Paul, Nesting-Induced Large Magnetoelasticity in the Iron-Arsenide Systems, Phys. Rev. B 90, 115102 (2014).

[53] H. Kontani and M. Ohno, Effect of a Nonmagnetic Impurity in a Nearly Antiferromagnetic Fermi Liquid: Magnetic Correlations and Transport Phenomena, Phys. Rev. B 74, 014406 (2006).

[54] H. Kontani, T. Saito, and S. Onari, Origin of Orthorhombic Transition, Magnetic Transition, and Shear-Modulus Softening in Iron Pnictide Superconductors: Analysis Based on the Orbital Fluctuations Theory, Phys. Rev. B 84, 024528 (2011).

[55] S. Mukherjee, A. Kreisel, P. J. Hirschfeld, and B. M. Andersen, Model of Electronic Structure and Superconductivity in Orbitally Ordered FeSe, Phys. Rev. Lett. 115, 026402 (2015); A. Kreisel, S. Mukherjee, P. J. Hirschfeld, and B. M. Andersen, Spin Excitations in a Model of FeSe with Orbital Ordering, Phys. Rev. B 92, 224515 (2015).

[56] S. Onari, Y. Yamakawa, and H. Kontani, Sign-Reversing Orbital Polarization in the Nematic Phase of FeSe Driven by Aslamazov-Larkin Processes, arXiv:1509.01172.

[57] K. Jiang, J. Hu, H. Ding, and Z. Wang, Interatomic Coulomb Interaction and Electron Nematic Bond Order in FeSe, Phys. Rev. B 93, 115138 (2016).

[58] D. S. Inosov, J. T. Park, P. Bourges, D. L. Sun, Y. Sidis, A. Schneidewind, K. Hradil, D. Haug, C. T. Lin, B. Keimer, 
and V. Hinkov, Normal-State Spin Dynamics and Temperature-Dependent Spin-Resonance Energy in Optimally Doped $\mathrm{BaFe}_{1.85} \mathrm{Co}_{0.15} \mathrm{As}_{2}$, Nat. Phys. 6, 178 (2010).

[59] N. Qureshi, P. Steffens, D. Lamago, Y. Sidis, O. Sobolev, R. A. Ewings, L. Harnagea, S. Wurmehl, B. Büchner, and M. Braden, Fine Structure of the Incommensurate Antiferromagnetic Fluctuations in Single-Crystalline LiFeAs Studied by Inelastic Neutron Scattering, Phys. Rev. B 90, 144503 (2014).

[60] Y. Nakai, S. Kitagawa, T. Iye, K. Ishida, Y. Kamihara, M. Hirano, and H. Hosono, Enhanced Anisotropic Spin Fluctuations below Tetragonal-to-Orthorhombic Transition in LaFeAs $\left(\mathrm{O}_{1-x} \mathrm{~F}_{x}\right)$ Probed by ${ }^{75}$ As and ${ }^{139} \mathrm{La}$ NMR, Phys. Rev. B 85, 134408 (2012).

[61] F. L. Ning, K. Ahilan, T. Imai, A. S. Sefat, M. A. McGuire, B. C. Sales, D. Mandrus, P. Cheng, B. Shen, and H.-H. Wen, Contrasting Spin Dynamics between Underdoped and Overdoped $\mathrm{Ba}\left(\mathrm{Fe}_{1-x} \mathrm{Co}_{x}\right)_{2} \mathrm{As}_{2}$, Phys. Rev. Lett. 104, 037001 (2010).

[62] L. Ma, G. F. Chen, D.-X. Yao, J. Zhang, S. Zhang, T.-L. Xia, and $\mathrm{W}$. Yu, ${ }^{23} \mathrm{Na}$ and ${ }^{75} \mathrm{As} N M R$ Study of Antiferromagnetism and Spin Fluctuations in NaFeAs Single Crystals, Phys. Rev. B 83, 132501 (2011).

[63] A. V. Chubukov, M. Khodas, and R. M. Fernandes, Magnetism, Superconductivity, and Spontaneous Orbital Order in Iron-Based Superconductors: Who Comes First and Why?, arXiv:1602.05503.

[64] H. Kontani and S. Onari, Orbital-Fluctuation-Mediated Superconductivity in Iron Pnictides: Analysis of the FiveOrbital Hubbard-Holstein Model, Phys. Rev. Lett. 104, 157001 (2010).
[65] A. E. Böhmer, F. Hardy, F. Eilers, D. Ernst, P. Adelmann, P. Schweiss, T. Wolf, and C. Meingast, Lack of Coupling between Superconductivity and Orthorhombic Distortion in Stoichiometric Single-Crystalline FeSe, Phys. Rev. B 87, 180505 (2013).

[66] T. Nomura, S. W. Kim, Y. Kamihara, M. Hirano, P. V. Sushko, K. Kato, M. Takata, A. L. Shluger, and H. Hosono, Crystallographic Phase Transition and High- $T_{c}$ Superconductivity in LaFeAsO:F, Supercond. Sci. Technol. 21, 125028 (2008).

[67] J. C. Slater, The Theory of Complex Spectra, Phys. Rev. 34, 1293 (1929).

[68] M. Aichhorn, S. Biermann, T. Miyake, A. Georges, and M. Imada, Theoretical Evidence for Strong Correlations and Incoherent Metallic State in FeSe, Phys. Rev. B 82, 064504 (2010).

[69] M. Aichhorn, L. Pourovskii, V. Vildosola, M. Ferrero, O. Parcollet, T. Miyake, A. Georges, and S. Biermann, Dynamical Mean-Field Theory within an Augmented Plane-Wave Framework: Assessing Electronic Correlations in the Iron Pnictide LaFeAsO, Phys. Rev. B 80, 085101 (2009).

[70] V. I. Anisimov, I. V. Solovyev, M. A. Korotin, M. T. Czyżyk, and G. A. Sawatzky, Density-Functional Theory and NiO Photoemission Spectra, Phys. Rev. B 48, 16929 (1993).

[71] L. Vaugier, H. Jiang, and S. Biermann, Hubbard $U$ and Hund Exchange J in Transition Metal Oxides: Screening Versus Localization Trends from Constrained Random Phase Approximation, Phys. Rev. B 86, 165105 (2012). 